\title{
Translating a Typical Business Process Modelling Language to a Web Services Ontology through Lightweight Mapping
}

\author{
Gayathri Nadarajan $^{1}$ Yun-Heh Chen-Burger ${ }^{2}$ \\ Centre for Intelligent Systems and their Applications (CISA) \\ School of Informatics, University of Edinburgh \\ Appleton Tower, Crichton St, Edinburgh EH8 9LE, Scotland \\ 1 Email: gaya.n@ed.ac.uk \\ http://homepages.inf.ed.ac.uk/s0450937/ \\ 2 Email: jessicac@inf.ed.ac.uk \\ http://www.aiai.ed.ac.uk/ jessicac/
}

\begin{abstract}
Bridging the gap between Enterprise Modelling methods and Semantic Web services is an important yet challenging task. For organisations with business goals, the automation of business processes as Web services is increasingly important, especially with many business transactions taking place within the Web today. Taking one approach to address this problem, a lightweight mapping between Fundamental Business Process Modelling Language (FBPML) and the Web Services Ontology (OWL-S) is outlined. The framework entails a data model translation and a process model translation via the use of ontologies and mapping principles. Several working examples of the process model translations are presented together with an implementation of an automated translator. FBPML constructs and process models that could not be translated to OWL-S equivalents highlight the differences between the languages of the two domains. It also implies that evolving Semantic Web technologies, in particular OWL-S, are not adequate for all service modelling needs and could thus benefit from the more traditional and mature BPM methods. On a more interesting note, this is effectively the first step towards enabling a semantic-based business workflow system.
\end{abstract}

Keywords: Enterprise Modelling, Business Process Modelling Methods, Semantic Web Services, FBPML, OWL-S, Ontologies, Lightweight Mapping.

\section{Introduction}

The need for more sophisticated Web-based support tools has become apparent with the fast advancement of the Web and the Semantic Web vision [5]. Business-to-Business (B2B) Electronic Commerce is fast becoming the most important application area of Semantic Web technology in terms of market volume [2]. Enterprise Modelling (EM) methods, on the other hand, are mature, established procedures that are commonly used as analysis tools for describing and redesigning businesses by entrepreneurs. They have been well recognised for their value in providing a more organised way to describe complex, informal domain [8].

The existence of established EM methods, such as Business Process Modelling (BPM) methods, suggests that 
they could be exploited by emerging technologies such as Semantic Web services to provide a more mature framework incorporating both business- and Web application-specific technologies. In a wider context this aims to bring business-oriented and technical-oriented communities closer in order to achieve common organisational goals.

To this end, several efforts have emerged to bridge the gap between EM methods and Semantic Web services. The notion of Semantic Business Process Management (SBPM) [13] was proposed to utilise semantic capabilities within business process technologies in one consolidated technology. This is motivated by the limited degree of mechanisation within BPM methods which could be supplemented by Semantic Web services frameworks. This effort complements the work presented by this paper. It also indicates an increasing interest in combining Semantic Web technologies with EM methods.

On a lower level, translation between languages could be categorised broadly into heavyweight and lighweight approaches. Heavyweight mapping takes into account the understanding of the semantics of the notations involved and provides a formalism for the mapping procedure. This approach is clearly more theoretical, however, recent work in mapping between BPM and Semantic Web languages have used a more pragmatic approach where identical or similar language constructs are mapped conceptually. FBPML has been translated to OWL-S in some preliminary work $[12,22,21]$, BPEL4WS has been mapped to OWL-S [30,4] and OWL-S has been mapped to WSMO [29]. Some initial work on mapping between ontologies has been carried out in [27], which proposes an abstract mapping language. This paper integrates the principles outlined in these efforts into a lightweight ontological conceptual mapping framework.

\subsection{Enterprise and Process Modelling}

The goal of applying an enterprise modelling method is to seek ways to improve an organisation's effectiveness, efficiency and profitability. EM methods are typically informal or semi-formal. They provide notations which enable entrepreneurs to describe aspects of their business operations. The notation is normally complemented with semi-formal or natural language descriptions which allows details of the business operations to be described.

Many EM methods have emerged to describe and redesign businesses, namely business process modelling, business system modelling and organisational context modelling methods [8]. Business Process Modelling (BPM) methods are able to formally express informally practiced procedures. More importantly, actions and effects of these processes can be demonstrated using simulation techniques. Some examples of BPM methods representations include Process Specification Language (PSL) [28], Integration DEFinition Language (IDEF3) [19], extended UML's Activity Diagram [26] and Petri-Nets [24].

In the course of half a decade ago, new process languages and models have been developed to promote the understanding and interoperability of process semantics over the Web, with the extensibility of operating over the Semantic Web. They are characterised by XML and XML-based languages, such as the Resource Description Framework (RDF) [15] and the Web Ontology Language (OWL). Some of these languages include Business Process Execution Language for Web Services (BPEL4WS), Business Process Modelling Language (BPML) [3], Web Service Ontology (OWL-S) [18], and more recently, Web Services Modelling Ontology (WSMO) [25].

\subsubsection{FBPML}

The Fundamental Business Process Modelling Language (FBPML) [9] is an inherited, specialised and combined version of several standard modelling languages. In particular, FBPML adapts and merges two established process languages; PSL and an IEEE standard, IDEF3. PSL provides formal semantics for process modelling concepts but does not provide visual notations or model development methods. IDEF3, on the other hand, provides visual notations and a rich modelling method, but its semantic is informal. FBPML is slightly adapted to provide a 
formal representation of IDEF3.

The main aim of FBPML is to provide support for virtual organisations which are becoming more and more pervasive with the advancement of Web technology and services. It ultimately seeks to provide distributed knowledgeand semantic-based manipulation and collaboration. Most importantly, people with different responsibilities and capabilities could work together to accomplish tasks and goals without technological or communication barriers caused by the differences in their roles.

Several characteristics of FBPML make it ideal as the BPM language of choice. It can express business processes in conventional first order predicate logic and has two sections to provide theories and formal representations for describing data and processes; the Data Language and the Process Language. It provides an abstraction that is separated from the actual implementation. Another ideal characteristic of FBPML is that it provides a visual presentation for the underlying ontology that is not present in most BPM languages. Visual data modelling languages may be Entity-Relationship and UML Data Diagram, among others. It also has a notion of time that may be synchronised.

FBPML has been applied successfully in several projects and tools, such as Knowledge Based Support Tool for Business Models (KBST-BM), Knowledge Based Support Tool for Enterprise Models (KBST-EM) ${ }^{1}$ and Advanced Knowledge Technologies (AKT) [1]. However, as with most BPM languages, FBPML could not be used directly with software systems, including web services.

\subsection{Emerging technologies for the Semantic Web}

The Semantic Web is a collaborative effort led by the World Wide Web Consortium (W3C) ${ }^{2}$ with participation from a large number of researchers and industrial partners. Since its introduction, the Semantic Web has become the buzzword among communities across many related disciplines; from knowledge engineers to human-computer specialists to business entrepreneurs. It is envisioned to be an extension of the current Web, in which information is annotated with meaning, so as to provide for better machine-processability and automated reasoning. Such provision, ultimately, would enable better cooperation between computers and people [5]. Being a cutting edge research field, some emerging technologies and standards are being developed to realise the Semantic Web. Three key languages are discussed and considered for the mapping framework.

\subsubsection{BPEL4WS}

Business Process Execution Language for Web Services (BPEL4WS) [6] is an industrial effort which formalises the specification of Web services, their flow composition and execution. By doing so, it extends the Web services interaction model and enables it to support business transactions. It defines an interoperable integration model that should facilitate the expansion of automated process integration within corporations and in business-to-business spaces. BPEL4WS builds on and extends XML and Web services specifications. The BPEL4WS process model is layered on top of several XML specifications, expressed entirely in XML, uses and extends Web Services Description Language (WSDL) [10], and uses WSDL and XML Schema to describe its data. However, using a representation such as XML Schema is inadequate for describing rich data models as required by Semantic Web services.

\subsubsection{WSMO}

The Web Services Modelling Ontology (WSMO) [25] provides a conceptual underpinning and a formal language for Semantic Web services. It has its conceptual basis in the Web Service Modelling Framework (WSMF) [11],

\footnotetext{
${ }^{1}$ KBST-BM and KBST-EM are developed by Artificial Intelligence Applications Institute (AIAI), University of Edinburgh.

${ }^{2}$ http://www.w3.org/
} 
refining and extending this framework and developing a formal ontology and set of languages. Its main modelling elements are Ontologies, Goals, Web services and Mediators and is described in the Web Service modelling Language (WSML) [17]. WSMO also has the aim of building editing tools and reference implementation. Current efforts $[16,29,23]$ have been focused on comparing and mapping OWL-S to WSMO, with the intention of incorporating the two technologies together. Although it promises an improvement to cost-effectiveness, scalability and robustness of current solutions, its framework is still very much in its early stages of development. Thus it could be premature for a concrete and realistic mapping to be performed with this language.

\subsubsection{OWL-S}

OWL-S [18] is a Web service ontology written in the Web Ontology Language (OWL) [20] extended with the Semantic Web Rule Language (SWRL) [14]. Its main aim is to describe Web services in machine-processable forms. OWL-S markup of Web services facilitates the automation of Web service tasks, including automated Web service discovery, execution, composition and interoperation. Following the layered approach to markup language development, the current version of OWL-S builds on OWL, which in turn, is built on top of the Resource Description Framework (RDF) [15], which is an XML-based framework that could be used to describe data models.

\subsection{Rationale}

BPEL4WS, although well accepted, has been primarily developed to execute Web services in that it works more like a programming language than a knowledge model or a process ontology. Therefore the semantics of processes written using BPEL4WS is not well-defined. Thus it does not provide a rich enough representation to support Semantic Web services as outlined in section 1.2.1. This brings OWL-S and WSMO as the closest competing technologies to be considered.

OWL-S has been criticised due to its relative simplicity and incompleteness, which has led to the development of WSMO. The conceptual and functional comparisons between the two [16] show that WSMO stands out as a more promising approach with its more extensive framework and support for ontological reuse and flexibility. It also enables the static and dynamic reuse of Web services to achieve the functionality of a more complex service. However, OWL-S is more mature in some aspects such as process model definition and the grounding of Web services since it has been under development for a longer time. Moreover, it has a distinct data and process model which makes it semantically richer and structurally similar to FBPML that allows for an appropriate mapping procedure to be carried out as will be outlined in the following sections. Therefore, although WSMO seems to be conceptually stronger than OWL-S, it has yet to further define some open aspects in order to be completely usable in real applications.

\section{Lightweight Ontological Mapping Framework}

A conceptual mapping framework was devised to translate FBPML to OWL-S, motivated by the fact that both languages have a clear separation between their data and process schemas. FBPML's data model is described in the FBPML Data Language while OWL-S is described in OWL and SWRL. FBPML's process model is described by the FBPML Process Language (FBPML PL), while OWL-S contains its own classes to describe its process model. Thus the mapping framework has been divided into a data model component and a process model component. The two clearly defined mapping parts constitute the main essence of this work.

The data and process models of each language are represented using ontologies. The ontology diagrams for FBPML data and process models were based on the Support Ontology from the AKT Project [1]. By using 
a semantic representation of the data and process models, the translation is conducted with accordance to the respective methodologies outlined in the following sections.

\section{Data Model Translation}

A data model describes how the relevant facts pertaining to a specific application is organised logically, namely the concepts, instances, relationships between them and their restrictions. The mapping of data models between FBPML and OWL-S involves the translation of representations of concepts (or classes), instances (of the concepts) and the relationships between the concepts and instances from FBPML DL to OWL. It also entails the translation of the restrictions (or constraints) from FBPML DL to OWL.

\subsection{FBPML Data Language}

The FBPML Data Language (FBPML DL) [7] is first-ordered. The syntactic convention that has been used in Pro$\log$ has been adopted for its representation. The four main parts that constitute FBPML DL are the foundational model, the core data language, the extension data language and the meta-predicates of FBPML. The foundational model includes the use of logic and set theory to represent concepts, primitive predicates and functions (relationships) and mathematical theory on the manipulation of integer, rational and real numbers. The core data language includes the fundamental predicates and functions that are common to all applications of the language while the extension data language contains predicates and functions that are additional to the core data language. They are usually user-defined and are often application and domain-specific. Meta-predicates give definitions for other predicates and may define axioms of an application model. The rest of this subsection provide an overveiw of the components of FBPML DL in Prolog syntactic convention where applicable.

Concepts. Concepts in the Foundational Model include the classes of things that could occur in a context or domain in question. The concepts include numbers (number/1), variables (variable/1), constants (constant/1), lists (list/1), strings (string/1) and terms (term/1).

Functions. Functions in FBPML DL include those that evaluate boolean expressions, such as if a term is an atom (atom/1), atomic (atomic/1), a compound (compound/1) or a list (is_list/1). There are functions that manipulate logical expressions such as not (not/1) and or (or/1), as well as arithmetic boolean expressions, such as the symbolic representations of equals $(=/ 2)$, greater than $(>/ 2)$, greater than or equals to $(>=/ 2)$, less than $(</ 2)$ and less than or equals to $(=</ 2)$.

Logical Quantification. FBPML provides for full first order logic expressiveness whereby quantification play an important role in determining the scope of the variables within a statement. Quantification expressions include forall (forall/1) and exist (exist/1). Constants true (true/0) and false (false/0) also provide for conditions that remain always true and false, respectively.

Predicates. The core predicates for the data language include classes, subclasses, instances, attributes, properties, constraints and relations; some which have been represented symbolically as functions, such as boolean arithmetic functions equal, greater_than, less_equal and so on. Table 1 contains the main predicates contained within the FBPML Data Language.

Meta-predicates. Meta-predicates define additional predicates to the core data language. Two such predicates include def_predicate(Pred, Def) and axiom(Conclusion, Hypothesis). Def_predicate introduces a 
Table 1. FBPML core predicates examples and their descriptions.

\begin{tabular}{|c|c|}
\hline Predicate & Description \\
\hline $\operatorname{class}(\mathrm{X})$ & $\mathrm{X}$ is a class; $\mathrm{X}$ is a string. \\
\hline subclass_of $(\mathrm{C} 1, \mathrm{C} 2)$ & $\mathrm{C} 1$ is a sublass of $\mathrm{C} 2$. \\
\hline instance $(\mathrm{X})$ & $\mathrm{X}$ is an instance (tangible or intangible) of the represented domain. \\
\hline property_name(Y) & $\mathrm{Y}$ is a property name for some classes. \\
\hline property_value(Z) & $\mathrm{Z}$ is a property value for a property of some classes. \\
\hline attribute_name(Y) & $\mathrm{Y}$ is an attribute name for some instances; $\mathrm{Y}$ is a string. \\
\hline attribute_value $(\mathrm{Z})$ & $\mathrm{Z}$ is an attribute value for a property of some classes; $\mathrm{Z}$ is a FBPML term. \\
\hline \multirow[t]{2}{*}{ class_property(C, P1, P2) } & Stores the property of class $\mathrm{C}$. \\
\hline & $\mathrm{P} 1$ is the property name, $\mathrm{P} 2$ is the property value. \\
\hline class_rel(R, C1, C2) & Defines the relationship between two classes. $\mathrm{C} 1$ holds the relationship $\mathrm{R}$ with $\mathrm{C} 2$. \\
\hline instance_of(I, C) & I is an instance of class $\mathrm{C}$. \\
\hline instance_att(I, A1, A2) & Stores an attribute value, A2, for attribute $\mathrm{A} 1$ of instance I. \\
\hline att_domain $(\mathrm{C}, \mathrm{A}, \mathrm{D})$ & $\mathrm{A}$ is an attribute for all instances in the class $\mathrm{C}$ for domain, $\mathrm{D}$. \\
\hline \multirow[t]{2}{*}{ instance_rel(R, I1, I2) } & Represents the relation $\mathrm{R}$ between two instances, I1 and I2. \\
\hline & $\mathrm{R}$ is a relationship that has been defined in class_rel( $\mathrm{R}, \mathrm{C} 1, \mathrm{C} 2)$. \\
\hline $\begin{array}{l}\text { static_constraint(Quantification, } \\
\text { Precondition, Conclusion) }\end{array}$ & Property that a valid goal state must have for a problem domain. \\
\hline $\begin{array}{l}\text { dynamic_constraint(Quantification, } \\
\text { Precondition, Conclusion) }\end{array}$ & Property that a goal state may have for a problem domain. \\
\hline equal $(\mathrm{X}, \mathrm{Y})$ & Returns true if $\mathrm{X}$ equals $\mathrm{Y}$. \\
\hline greater_than $(\mathrm{X}, \mathrm{Y})$ & Returns true if $\mathrm{X}$ is greater than $\mathrm{Y}$. \\
\hline greater_equal $(\mathrm{X}, \mathrm{Y})$ & Returns true if $\mathrm{X}$ is greater than or equals to $\mathrm{Y}$. \\
\hline less_than $/ 2$ & Returns true if $\mathrm{X}$ is less than $\mathrm{Y}$. \\
\hline less_equal/2 & Returns true if $\mathrm{X}$ is less than or equals to $\mathrm{Y}$. \\
\hline
\end{tabular}

new predicate, Pred and its definition, Def from exisitng predicates, while axiom defines properties of existing predicates.

\subsection{OWL-S Data Language: OWL}

OWL [20] is a W3C recommendation for a Web ontology language and is the representation language for OWL-S ontologies. One reason OWL was chosen as the representation language for OWL-S is that it is compatible with $\mathrm{XML}$ and RDF while providing additional expressiveness thus allowing users to formally describe more types of classes, properties, individuals, and relationships than XML or RDF. OWL provides three increasingly expressive sublanguages: OWL Lite, OWL Description Logic (OWL DL), and OWL Full. OWL Lite provides the least expressive power among the three and thus was not expressive enough for OWL-S needs whereas OWL Full contains all the OWL language constructs and provides free, unconstrained use of RDF constructs but is undecidable. OWL DL provides maximum expressiveness while retaining computational completeness and decidability and thus lays the foundation for efficient reasoning support for the Semantic Web. Although OWL adds considerable expressive power to the Semantic Web, it does have limitations, particularly with respect to expressing rules. Therefore it has been extended with the Semantic Web Rule Language (SWRL) to overcome these limitations. The rest of this subsection provides an overview of the main constructs in OWL. 
Classes and Instances. Classes are defined using the owl:Class element. There are two predefined classes, owl:Thing, which defines the most general class that contains everything, and owl:Nothing, which is the empty class. Hence, every class is a subclass of owl: Thing and a superset of owl: Nothing. Instances, as in FBPML and many conventional programming languages, are actual occurrences of the classes or objects that in turn represent an abstraction for them. The syntax for OWL class and instance could be found in Table 2.

Property Elements and Restrictions. Two kinds of properties are relevant to OWL; object properties and data type properties. An object property relates (all) instances of a class to (all) instances of another class while a data type property relates (all) instances of a class to datatype values, such as integers, strings and so on. OWL also expresses built-in properties such as transitivity (owl:TransitiveProperty), symmetry (owl: SymmetricProperty) and inverse (owl: inverse0f). One can specify constraints or restrictions on properties using the rdfs: subClassOf element. In general, an owl:Restriction element contains an owl:onProperty element and one or more restriction declarations.

Quantification and Cardinality Restrictions. The elements owl:allValuesFrom and owl:someValuesFrom provide for logical universal and existential quantification respectively. However, the notion of quantification within OWL is still limited and is being extended by other logical formalisms such as SWRL. Cardinality restrictions can also be expressed using similar conventions for property restrictions, the restriction declaration would contain owl:minCardinality and/or owl:maxCardinality elements to define the range of cardinality allowed.

Boolean Combinations. Union, intersection and complement of classes are some binary relations that can be expressed directly in OWL-S using the constructs owl:unionOf, owl:intersectionOf and owl:complement0f elements. Other boolean combinations are also possible. For example, if we want to express that "a is disjoint with b", the following syntax could be used:

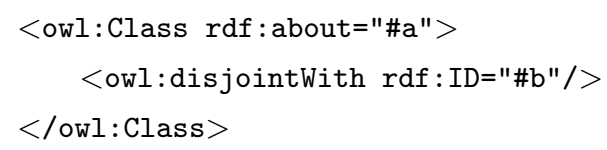

Data types. OWL does not have any predefined data types, but it allows one to use XML Schema's predefined data types. Although XML Schema provides for a mechanism to construct user-defined data types, these cannot be used in OWL. Furthermore, only some of the predefined data types can be used in OWL, such as string, integer, Boolean, time and date. It could be argued that the use of data types within OWL is still limited.

\section{Data Model Mapping}

The devised data model translation encompassed the mapping of classes, instances, relationships and restrictions. The procedure for data model mapping could be summarised in three main steps, refined from [29].(1) Pre-processing (if any), for instance organise modelling constructs into Prolog readable syntax. (2) Mapping of ontologies, the two ontologies in question are provided by Figures 3 and 4. This consists of the following steps. (i) Mapping of classes, which includes the mapping between the concepts (ii) Mapping of instances, which is the mapping of the instantiation of the concepts (iii) Mapping of relationships between the classes, instances and attributes and (iv) Mapping of restrictions which entails the mapping of constraints between the data models. (3) Mapping for rules/axioms. 
The remaining subsections elaborate these steps, provide the mapping using an adaptation of the formal mapping language initiated by [27] and the syntax of the translations between the data models of FBPML and OWL.

\subsection{Ontologies of the Data Models}

The data model of FBPML and OWL-S are represented semantically using ontology notation, as described by Figure 3 and Figure 4. Applying the mapping principles to the derived ontologies, a class in FBPML is mapped to a class in OWL-S, an instance of a class in FBPML is mapped to an instance of a class in OWL-S and a relationship in FBPML is mapped to a relationship in OWL-S. The following subsection provides the formalism for the mapping and the translated syntax from FBPML DL to OWL.

\subsection{Mapping of Classes and Instances}

Following the Semantic Web layering approach, OWL is an RDF-based language that utilises tags and tree-like structures for its representation, whereas FBPML DL is first-ordered. In the following section, syntax for classes, instances and relations in FBPML DL and their corresponding translations in OWL are provided. The mapping between the classes (concepts) and instances (objects) could be done directly, following the formalism provided below and as illustrated by Table 2 .

classMapping (unidirectional, <fbpml_dl, concreteClass>, <owl, class>).

classMapping (unidirectional, <fbpml_dl, abstractClass>, <owl, class>).

classMapping (unidirectional, <fbpml_dl, instanceofClass>, <owl, instanceofClass>).

Table 2. Mapping of classes and instances between FBPML DL and OWL

\begin{tabular}{|c|c|c|}
\hline Concept & FBPML & OWL \\
\hline Concrete & concrete_class(Name, & <owl:Class rdf:ID="ClassName"> \\
\hline \multirow[t]{2}{*}{ Class } & Description,Example, Rules, & $<$ rdfs $:$ comment $>$ A comment $</$ rdfs $:$ comment $>$ \\
\hline & CrossReferences,ObjectAttributes) & $<$ /owl:Class $>$ \\
\hline Abstract & abstract_class(Name, & <owl:Class rdf:ID="AbstractClassName" > \\
\hline \multirow[t]{2}{*}{ Class } & Description,Example,Rules, & $<$ rdfs $:$ comment $>$ A comment $</$ rdfs $:$ comment $>$ \\
\hline & CrossReferences, ObjectAttributes) & $</$ owl:Class $>$ \\
\hline Instance & instance_of(InstanceName, & $<$ ClassName rdf:ID="Instance Name" $>>$ \\
\hline & ClassName) & \\
\hline
\end{tabular}

\subsection{Mapping of Relationships}

Binary relationships can be divided into three types in the context of the data model translation; those between two classes, those between two instances of a class and those between an instance of a class and a class. As some languages prefer to use certain conventions for specification, it should be clarified that properties are relationships, just as objects are instances. For example, OWL specifies relations using the elements owl:DatatypeProperty and owl:ObjectProperty. Thus an instance-to-instance relationship in FBPML (instance_rel/3) is translated to an object property in OWL while an instance-to-class relationship in FBPML (instance_att/3) is mapped to an OWL datatype property. A class relationship in FBPML is mapped to a class property in OWL. OWL has a predefined set of class 
properties such as "disjointWith", "equivalentClass" and so on. Table 3 provides the syntax for the translation of relationships from FBPML DL to OWL. Formally this could be expressed as follows:

classMapping (unidirectional, <fbpml_dl, classRelationship>, <owl, classProperty>).

classMapping (unidirectional, <fbpml_dl, instanceRelationship>, <owl, objectProperty>) .

classMapping(unidirectional, <fbpml_dl, instanceAttribute>, <owl, datatypeProperty>) .

Table 3. Mapping of relationships between FBPML DL and OWL

\begin{tabular}{|c|c|c|}
\hline Relationship & FBPML & OWL \\
\hline Class-to-Class & class_rel(Relation, & <owl:Class rdf:about="\#Class1"> \\
\hline Relationship & Class1,Class2) & $\begin{array}{l}<\text { owl:Relation rdf:resource="\#Class2"/> } \\
</ \text { owl:Class }>\end{array}$ \\
\hline $\begin{array}{l}\text { Instance-to-Instance } \\
\text { Relationship }\end{array}$ & $\begin{array}{l}\text { instance_rel(Relation, } \\
\text { Instance1,Instance2) }\end{array}$ & $\begin{array}{l}\text { <owl:ObjectProperty rdf:ID="Rel"> } \\
<\text { rdfs:domain rdf:resource="\#0bj1"/> } \\
\text { <rdfs:range rdf:resource="\#0bj2"/> } \\
<\text { /owl:ObjectProperty }>\end{array}$ \\
\hline $\begin{array}{l}\text { Instance-to-Class } \\
\text { Relationship }\end{array}$ & $\begin{array}{l}\text { instance_att(Instance, } \\
\text { AttributeName,AttributeValue) }\end{array}$ & $\begin{array}{l}<\text { owl:DatatypeProperty } \mathrm{rdf}: \mathrm{ID}=" \mathrm{Rel} " /> \\
<\text { rdfs:domain } \mathrm{rdf}: \text { resource="\#Obj1"/> } \\
\text { <rdfs:range rdf:resource="\&xsd;string"/> } \\
<\text { /owl:DatatypeProperty }>\end{array}$ \\
\hline
\end{tabular}

The mapping procedure for relationships is slightly more complicated, as FBPML DL does not have a separate predicate to explicitly describe a particular relationship, whereas OWL provides elements to differentiate between object and datatype properties. Thus, to perform the translation, one has to extract the relationship name from the class or instance relationship. An obvious difference between FBPML DL and OWL is that in OWL, all distinct objects (classes, instances, data types, etc) have unique Uniform Resource Identifiers (URI), which avoids ambiguity when referring to objects with the same name over the Web but may not be equivalent. FBPML is a BPM language which is, at present, not directly compatible with Web services, thus it does not support URI based naming conventions. However, a default URI (such as a project's local URL) could be used as the base URI when the translation takes place.

\subsection{Mapping of Restrictions}

Restrictions in OWL are expressed as constraints in FBPML DL. In FBPML DL, two types of constraints are provided, static and dynamic constraints. The syntax for these predicates are provided in Table 1. A static constraint is a hard constraint. As constraints are often domain- and application-dependent, they are only meaningful when associated to a particular problem domain. Dynamic constraints are soft constraints that may or may not be obeyed by the desirable goal states for the problem domain. In other words, static constraints must be satisfied while dynamic contraints are desirable for an application. In OWL, all the resrictions are hard constraints. Therefore a FBPML static constraint is mapped to an OWL restriction. Formally this mapping is expressed as:

classMapping(unidirectional, <fbpml_dl, staticConstraint>, <owl, restriction>).

For the example, the constraint "All first year courses must be taught by a professor" could be translated from FBPML to OWL in the following syntax. 
FBPML DL:

static_constraint([forall(X), forall(Subclass)], [subclass_of(Subclass, firstYearCourse), instance_rel(isTaughtBy,

Subclass, $\mathrm{X})],[\mathrm{X}=$ professor $])$.

OWL translated syntax:

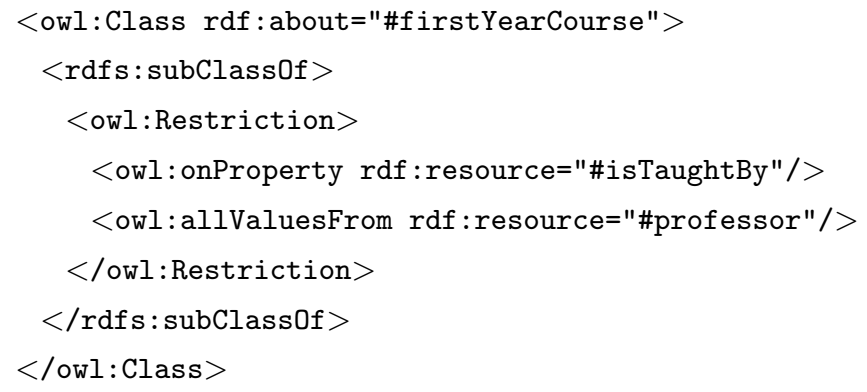

Using a similar approach, restrictions with existential quantification could also be expressed in both languages and mapped accordingly using the exist/1 predicate and owl : someValuesfrom element respectively.

The mappings provided entail those between concepts in the two ontologies which are most significant for the data model mapping. However there are also mappings between the relations in the ontologies that could be formalised as follows:

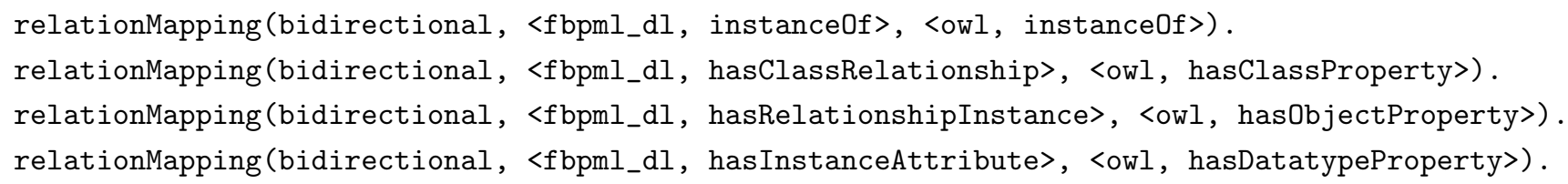

\section{Process Model Translation}

A process model essentially describes how a process or task is done. The process model translation entailed the mapping between FBPML PL and OWL-S.

\subsection{FBPML Process Language}

FBPML has a visual representation of its process models, which makes it intuitive for humans to understand the execution of processes described by it. This is adapted from the IDEF3 process language, which provides a rich diagrammatic representation for its process models. Apart from that, it also supports full first order predicate logic. Thus, it provides powerful and expressive semantics for describing process execution.

\subsubsection{Main Process Model Components}

In FBPML, the terms process, activity and task are used interchangeably. A model described in FBPML is made up of Main Nodes, Junctions, Links and Annotations. Some of the process elements of FBPML PL are contained in Figure 1.

Main Nodes. Activity is the main concept to denote a process which may be further decomposed or specialised into subprocesses. The three main components of an activity are Trigger(s), Preconditions, and Action(s). Primitive Activity is a leaf node activity that may not be further decomposed or specialised. Primitive activities are directly connected to application layers. 
Role is a specialised function, capability or authority possessed by an enabler ${ }^{3}$ over a set of activities, i.e. a responsibility in context. Time Point is used to express temporality and indicates a particular point in time during the process execution. Graphically time points are represented as the circular ends of either sides of a Synchronisation Bar.

Links. Links between processes consist of Precedence Links and Synchronisation Bars which place temporal constraints on process execution. Precedence Link is comparable to the more constrained Precedence Link, type II, in IDEF3. It indicates the latter activity cannot start until the former has finished. Synchronisation Bar also places a temporal constraint between two time points. This notation enables any time points to be made equivalent and therefore enables process operations to be synchronised, or executed concurrently.

Junctions. Junctions are used to connect multiple activities. They also define the temporal constraints and control the initiation and finishing of parallel processes. The four types of Junctions in FBPML are Start, Finish, And and Or. Each use of a junction is a type of one-to-many (split junction) or many-to-one (joint junction) relationships. The two most commonly used junctions in the split and joint contexts are And and Or. A subset of the Or construct is the Xor, which imposes that only one process is selected for execution. Their semantics are equivalent to the logical connectives 'And', 'Or' and 'Xor', respectively.

And-Joint or Or-Joint indicates that there is more than one process preceding the And or Or junction but there is only one process following the junction. Semantically, an And-Joint indicates the process execution flow and the temporal constraint that all of the preceding activities must be finished before the following activity is temporally qualified and therefore be executed. And-Split or Or-Split means that only one process will proceed to the And or Or junction, but more than one process will follow the junction. An And-or Or-Split indicates that all of the following activities become temporally qualified when the preceding activity is finished. Furthermore, an And-Split also indicates that all of the following activities must be executed at some point of time after the preceding activity is finished. Start and finish denote the commencement and completion of a process model execution. Section 6.2.1 provides an example process diagram and formal description of one of these constructs. Combinations of $A n d-$ And, And-Or, Or-And and Or-Or constructs are also used to represent more complicated models. Section 6.2.2 illustrates an example that contains the And-And construct.

Annotations. Annotations include Idea Note and Navigation Note. Neither of them contribute to the formal semantics of the process model. Instead, they are used to help users to understand the processes more clearly from an intuitive point of view. Idea Note records information which is related to the processes but not part of the process model. Navigation Note records the relationships between diagrams in a model.

Action/Process Decomposition. Task decomposition allows a process described at a higher level of abstraction to be decomposed into more detailed sub-processes that are more explicit for its implementation procedures. Alternative decomposition defines alternative ways of decomposing a higher level process into different sets of subtasks; where only one set of those sub-tasks needs to be finished to finish the higher level process.

\subsubsection{Additional Primitives}

Apart from the primitives described above, FBPML also provides some additional primitives such as time and its manipulation. Trigger is a related construct that possesses full first-order logic expressiveness and thus could express temporal behaviour adequately. It also describes conditions and rules very well. Preconditions and Postconditions

\footnotetext{
${ }^{3}$ An individual, a group of people or a software component
} 
are used in conjunction with conditional statements that are equivalent to if-then-else constructs in conventional programming terms. FBPML also describes event and process life status and cycles. Each node (or process) in FBPML has its attributes, such as Process: Instance_Id, Process_type, Life_status, Priority, Average_time_cost, Begin/End_time, Service_Requester/Service_Provider, Trigger, Preconditions, Actions, Postconditions.

\title{
5.1.3 FBPML Formal Definition
}

While the FBPML graphical representation provides intuitive reasoning, the FBPML formal definition allows for machine processability. The following definition has been extracted from $[9]^{4}$ :

\author{
Main Nodes: \\ activity(ID, Name, Trigger, Precondition, Postcondition, Action, Description). \\ primitive_activity(ID, Name, Trigger, Precondition, Postcondition, Action, Description).
}

\section{Action:}

action(ActionType, Class, Instance).

\section{Junctions:}

start(ActivityName).

junction(JunctionType, PreActivities, PostActivities).

\section{Links:}

link(PrecedingActivity, ProceedingActivity).

syn( begin/end(Activity1), begin/end(Activity2) $)^{5}$.

\subsection{OWL-S Process Components}

OWL-S is made up of four main ontologies or classes; Service, Service Profile, Service Model and Grounding. The basic properties that describe an OWL-S process are Input, Output, Precondition and Effects. For the purpose of the process model mapping, attention is given to a subclass of Service Model, the class Process, which most appropriately defines the execution of a process.

\subsubsection{Process}

The class Process draws upon well-established work in a variety of fields, including work in AI on standardisations of planning languages, work in programming languages and distributed systems, emerging standards in process modelling and workflow technology such as Process Specification Language (PSL) and the Workflow Management Coalition effort [31], work on verb semantics and event structure, previous work on action-inspired Web service markup, work in AI on modelling complex actions and work in agent communication languages. Subclasses of Process are Atomic Process, Simple Process and Composite Process.

Atomic Processes are directly invocable, have no subprocesses and execute in a single step. They take an input message, process it, and then return their output message. For each atomic process, there must be provided a

\footnotetext{
${ }^{4}$ The initial definition for activity $/ 6$ and primitive_activity $/ 6$ have been extended to activity $/ 7$ and primitive_activity $/ 7$ by the inclusion of Description.

${ }^{5}$ This is a shorthand for the four possible combinations: syn(begin(Activity1), begin(Activity2)), syn(begin(Activity1),end(Activity2)), $\operatorname{syn}($ end(Activity1), begin(Activity2)) and syn(end(Activity1), end(Activity2)).
} 
grounding that enables a service requester to construct messages to the process from its inputs and deconstruct replies.

Simple Processes are not invocable and are not associated with a grounding, but, like atomic processes, they are conceived of as having single-step executions. Simple processes are used as elements of abstracton; a simple process may be used either to provide a view of some atomic process, or a simplified representation of some composite process (for purposes of planning and reasoning).

Composite Processes are decomposable into other (non-composite or composite) processes. A precise specification of what it means to perform a process has not yet been given, but it applies that if a composite is a Sequence, then the client sends a series of messages that invoke every step in order. One crucial feature of a composite process is its specification of how its inputs are accepted by particular subprocesses, and how its various outputs are produced by particular subprocesses.

\subsubsection{Parameter and Result}

Parameter stores inputs, outputs and results. It is a subclass of the SWRL's swrl:Variable construct with a type description and an optional value expression, whereby the value of a constant parameter could be stored, for instance. Input, Output, Local and ResultVar are subclasses of Parameter. Local Parameters may only be used with Atomic Processes. Their function is to identify variables whose scope is the process as a whole, so that when they are bound in preconditions, they can be referenced in outputs and effects. Outputs are process parameters that are associated with descriptions by OutputBindings associated with particular result conditions. Result has zero or more inCondition properties, withOutput properties, hasResultVar properties and hasEffect properties.

\subsubsection{Control Constructs}

Control constructs govern the flow of execution of a process. Control constructs make up a composite process each composite process is composedOf control constructs, thus they play an important role in characterising an OWL-S web service. The following are descriptions of the major control constructs in OWL-S.

A Sequence is a list of control constructs to be done in order. Generally, process models that are composed of a series of subprocesses are enclosed within a sequence construct in OWL-S.

The components of a Split process are a bag of process components to be executed concurrently. Split completes as soon as all of its component processes have been scheduled for execution, which means that all processes within the Split construct bag must start for this control construct to terminate successfully. Split-Join is used when a process consists of concurrent execution of a bunch of process components with barrier synchronisation. Split-Join completes when all of its components processes have completed. Note that this control construct complements the Split control construct.

Choice calls for the execution of a single control construct from a given bag of control constructs. Any one of the given control constructs can be chosen for execution. Note that this is similar to the Split control construct except that it imposes that only one process is selected for execution over a bag of processes. The Any-Order control construct allows the process components to be executed in some unspecified order but not concurrently. All components are required to execute and complete. All components must be executed, and as with Split-Join, completion of all components is required.

If-Then-Else has properties ifCondition, then and else, which correspond to the If, Then, Else constructs in conventional programming language terms.

The Iterate construct does require the number of iterations to be specified; the initiation, termination or maintenance could be specified with the whileCondition or an untilCondition. It is defined to serve as the common superclass of Repeat-While, Repeat-Until and potentially other specific control constructs that might 
be needed in the future. Repeat-While and Repeat-Until control constructs iterate until a condition becomes false or true, as in most programming language conventions. Thus, Repeat-While may never act, whereas Repeat-Until always acts at least once.

\subsubsection{Expression}

Expression contains ontology elements for capturing conditions and effects, it is the superclass of Condition and Effect. Conditions, (preconditions in FBPML terms), is defined as a class of logical expressions, whose truth value can be determined for further action, for instance it could be used with the If-Then-Else control construct. Expressions can be in any allowed logical language, and use parameters (primarily input and local) variables. Logical expressions for preconditions, effects and result conditions are represented in a logical formalism. SWRL has been the choice of extension for OWL due to its well formed semantics.

\subsection{Ontologies of the Process Models}

Figure 6 describes the process model ontology for FBPML. Note that the Model Construct concept is not part of the FBPML PL notation, but it is added to the ontology to assist the mapping effort. As the process models are more extensive, the primitives are mapped first before applying mapping principles for process model translation.

\section{Process Model Mapping}

The primitives in FBPML PL were mapped to the primitives in OWL-S. The formal mapping of the primitives is given below:

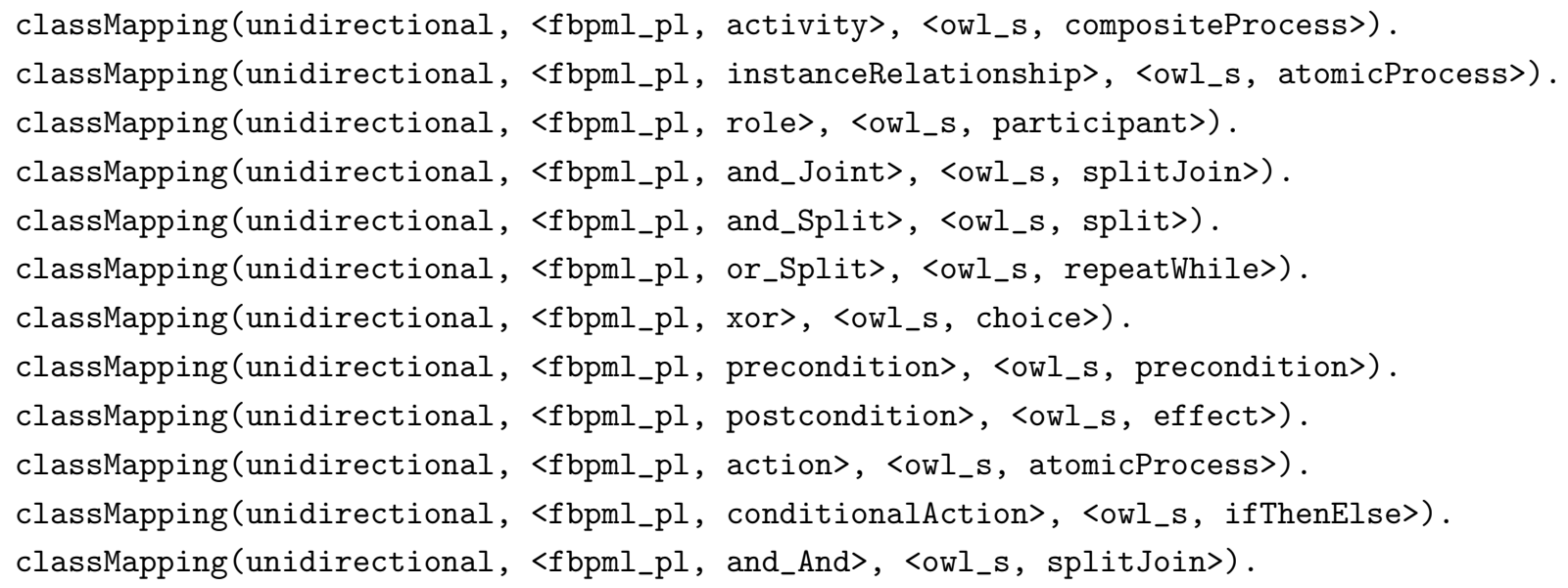

Several observations were made from the mapping of primitives between their process elements. In FBPML, a role is a responsibility in context; usually in the form of a Provider or a Requester. In OWL-S the Participant instances include TheClient and TheServer. Thus the Requester role corresponds to TheClient in OWL-S and the Provider role corresponds to TheServer OWL-S. FBPML's notion of role is richer and could refer to an individual, a group of people or software components or a combination of the above [9].

The time ontology is used currently in limited ways in the process specification in OWL-S (described in http://www.isi.edu/ pan/damltime/time-entry.owl). Although there is no OWL-S construct that maps to FBPML's Precedence Link, the order of execution of processes within a sequence enclosed by <objList:first> ..</objList:first> and <objList:rest>.. </objList:rest>. 
Table 4. Summary of mapping between FBPML and OWL-S process primitives

\begin{tabular}{|c|c|c|c|c|c|}
\hline Primitive & FBPML & OWL-S & Primitive & FBPML & OWL-S \\
\hline \multirow[t]{4}{*}{ Main Nodes } & Activity & Composite Process & Annotations & Idea Note & See Note below \\
\hline & Primitive Activity & Atomic Process & & Navigation Note & See Note below \\
\hline & Role & Participant & Process & Precondition & Precondition \\
\hline & Time Point & See Note & Components & Trigger & See Note below \\
\hline \multirow[t]{2}{*}{ Links } & Precedence Link & (part of) Sequence & & Postcondition & Effect/Output \\
\hline & Synchronisation Bar & See Note & & Precondition, Trigger & Input \\
\hline \multirow[t]{6}{*}{ Junctions } & Start & See Note & & Action & Atomic Process \\
\hline & Finish & See Note & & Conditional Action & If-Then-Else \\
\hline & And-Joint & Split-Join & Combination & And-And & Split-Join \\
\hline & Or-Joint & See Note & Model & And-Or & See Note below \\
\hline & And-Split & Split & Constructs & Or-And & See Note below \\
\hline & Or-Split & Repeat-While/Until & & Or-Or & See Note below \\
\hline
\end{tabular}

Note: Limited (or no) equivalent convention provided by OWL-S.

The And-Split is slightly modified (with barrier synchronisation) to correspond to the OWL-S Split construct where the processes that branch into the junctions are synchronised. The OWL-S Choice construct selects one process out of many for execution, which is equivalent to the Xor in FBPML, however, OWL-S does not provide a direct equivalent for the Or construct. The Xor junction is subsumed by the Or junction and has been utilised by more recent applications to make it more explicit for automation when bridging to (Semantic Web or Web service) methods that do not have Or junctions.

Since OWL-S does not support the notion of trigger, the combination of Precondition and Trigger in FBPML approximately map to Precondition in OWL-S. Postcondition in FBPML describes the effects and conditions which must hold true after the execution of a process.

In FBPML, an Action is the actual execution behaviours in a process model which can be stored in a repository. The advantage of the action repository is that actions can be reused and shared. Therefore an Action approximately maps to OWL-S Atomic Process. A precise specification of what it means to perform a process in OWL-S has not been given yet. Table 4 summarises the mapping of the primitives between FBPML PL and OWL-S process ontology.

\subsection{Process Model Mapping Principles}

After determining the matching primitives, the mapping was followed through using component models consisting of sequences, and junctions. The procedure for the mapping of component models involved breaking down each process into a composite process, and considering a single construct to be translated, such as Sequence, Split, Split-Join or Choice. The whole composite process consists of a sequence of at most two processes. This component model translation could be incrementally extended to cater for more complex models via a layered model translation. When the procedure is refined to cater for all process models, a general methodology for the process model mapping was achieved (refined from [12]).

1. Decompose FBPML process model in a top-down order. 


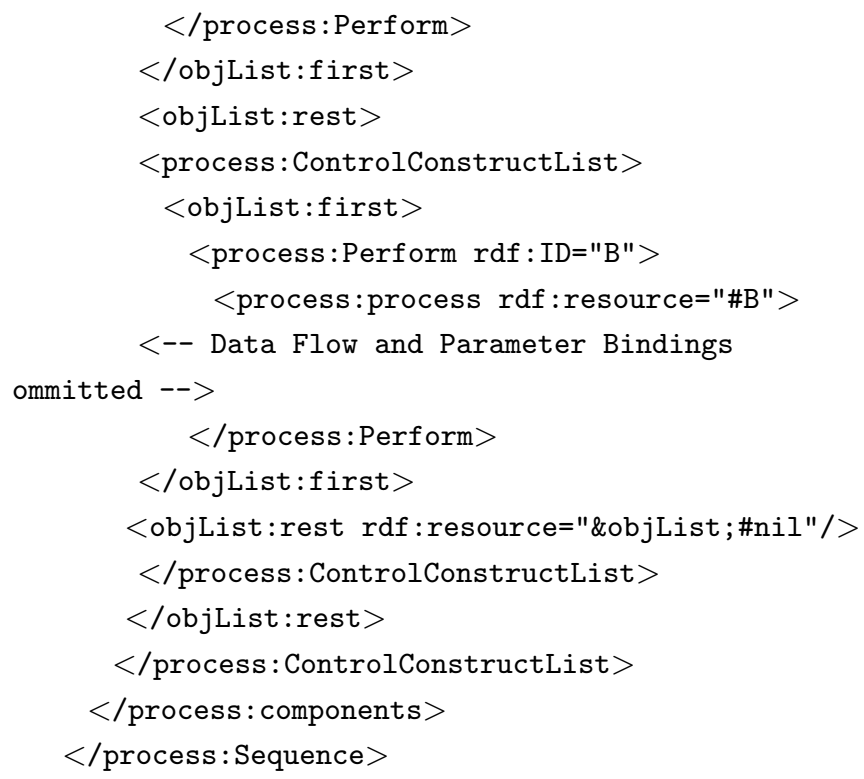

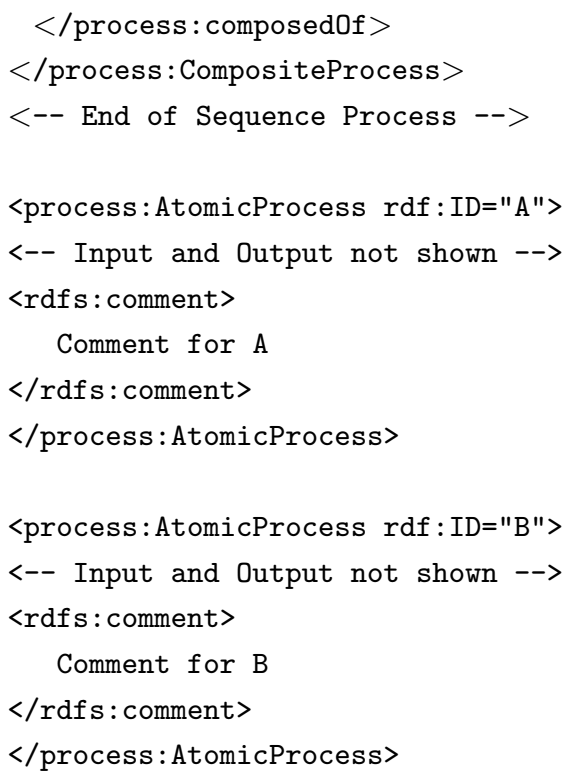

$</$ process:AtomicProcess $>$

\section{Example 2. And-Joint}

FBPML formal notation for process model in Figure 9: activity(02, 'And-Joint Construct', Trigger, Precondition, Postcondition, Action, Description). primitive_activity(003, 'A', Trigger, Precondition, Postcondition, Action, 'Comment for A'). primitive_activity(004, 'B', Trigger, Precondition, Postcondition, Action, 'Comment for B'). primitive_activity(005, 'C', Trigger, Precondition, Postcondition, Action, 'Comment for $\left.C^{\prime}\right)$. junction('And', ['A', 'B'], 'C').

Assumptions and Interpretations:

- $\mathbf{C}$ cannot be started unless both $\mathbf{A}$ and $\mathbf{B}$ have finished executing. (indicated by the And-Joint junction).

- The whole junction is taken to be a composite process $^{9}$.

Following the same approach applied to the previous example, the process is defined to be a Composite Process in OWL-S. The general algorithm for the translation is given in Algorithm 1. For this example, a detailed algorithm of step 6 of Algorithm 1 could be given as follows:

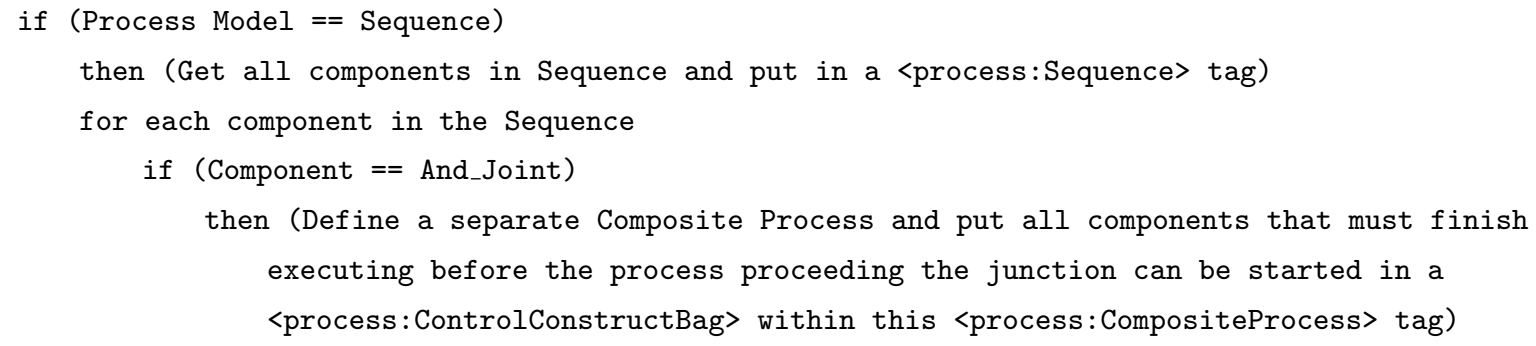

The OWL-S syntax for the translated sequence and atomic processes are the same as those provided in the previous

\footnotetext{
${ }^{9}$ In reality the junction should be preceded by another (split) junction or other processes preceeding A and B, but it is represented on its own in this example for demonstration purposes
} 
Sequence example. This junction is equivalent to the OWL-S Split-Join construct, a similar representation of the use of the Split-Join construct is provided by the And-And junction.

A complement component to the And-Joint construct is the And-Split. Translated to OWL-S, it is also composed of a sequence of two composite processes, but a primitive activity is followed by an And-Split process. This junction is approximately equivalent to the Split construct in OWL-S.

Another FBPML process component that has been translated is the Xor-Split. This subset of the Or-Split construct is used because no equivalent construct for Or is found in OWL-S. The the Xor-Split junction is equivalent to the Choice control construct in OWL-S, this mapping is direct. This translation is identical to the that of the And-Split except that it corresponds to the OWL-S Choice control construct.

\subsubsection{More Complex Models}

More complex process models include the use of combinational branching junctions; the four basic cases of these junctions are And-And, And-Or, Or-And and Or-Or. Since OWL does not provide for the $O$ control construct, the only coupled junction that can be mapped is the $A n d-A n d$ and is illustrated below.

\section{Example 1. Combinational And-And Junction}

FBPML formal notation for process model in Figure 10:

activity(06, 'And-And Junction', Trigger, Precondition, Postcondition, Action, 'Desc').

junction('And', 'A', ['B', 'C', 'D']).

junction('And', ['B', 'C', 'D'], 'E').

primitive_activity(001, 'A', Trigger, Precondition, Postcondition, Action, 'Comment for A').

primitive_activity(002, 'B', Trigger, Precondition, Postcondition, Action, 'Comment for B').

primitive_activity(003, 'C', Trigger, Precondition, Postcondition, Action, 'Comment for C').

primitive_activity(004, 'D', Trigger, Precondition, Postcondition, Action, 'Comment for D').

primitive_activity(005, 'E', Trigger, Precondition, Postcondition, Action, 'Comment for E').

The And-And junction utilises the combination of the And-Split and And-Joint junctions. Following the methodology provided in section 6.1, the process model contained in this figure could be decomposed as shown in Figure 11.

Taking the And-And junction as an OWL-S Split-Join, the order of execution is given by the prefix notation: Sequence (A, Split-Join(B,C,D), E).

The translated syntax in OWL-S as generated by the automatic translator:
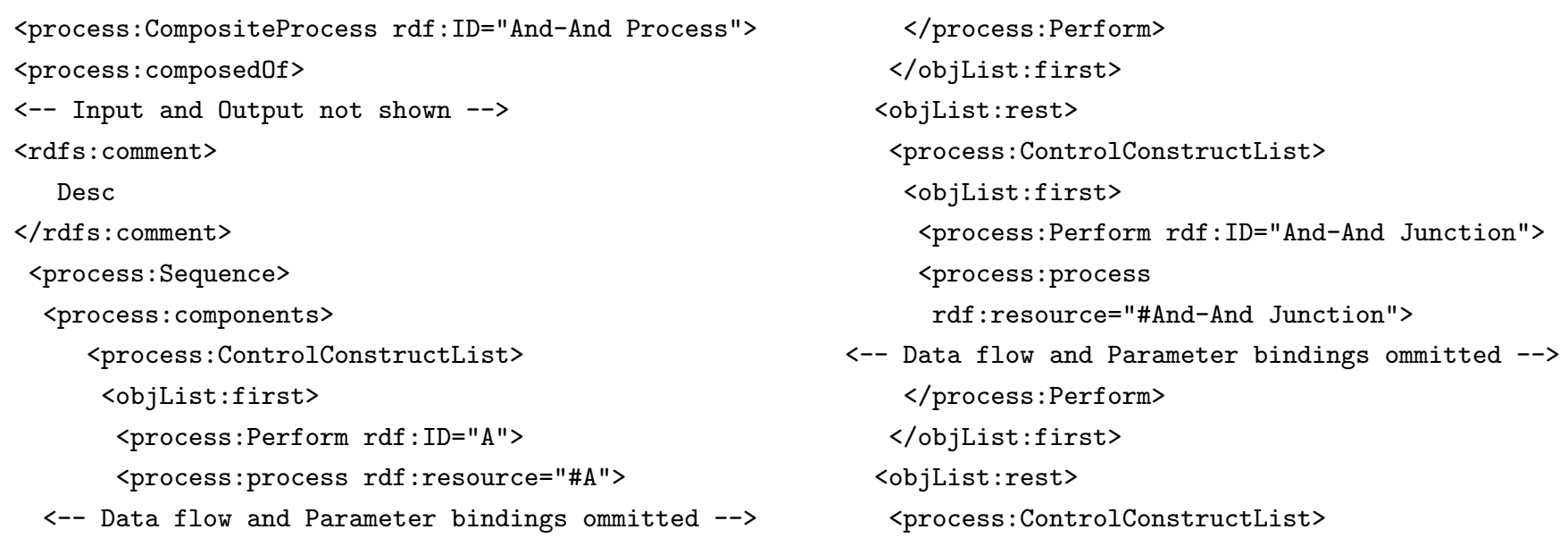


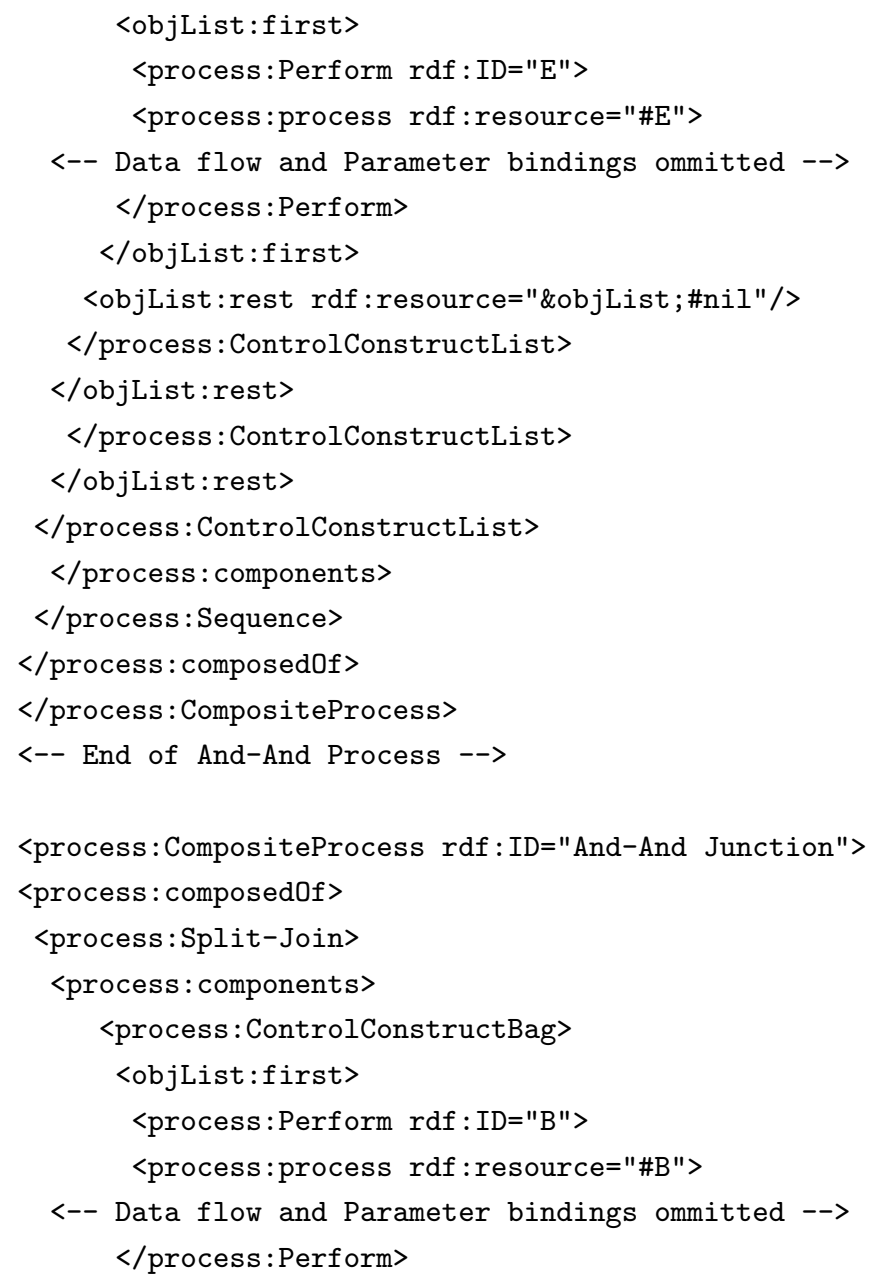

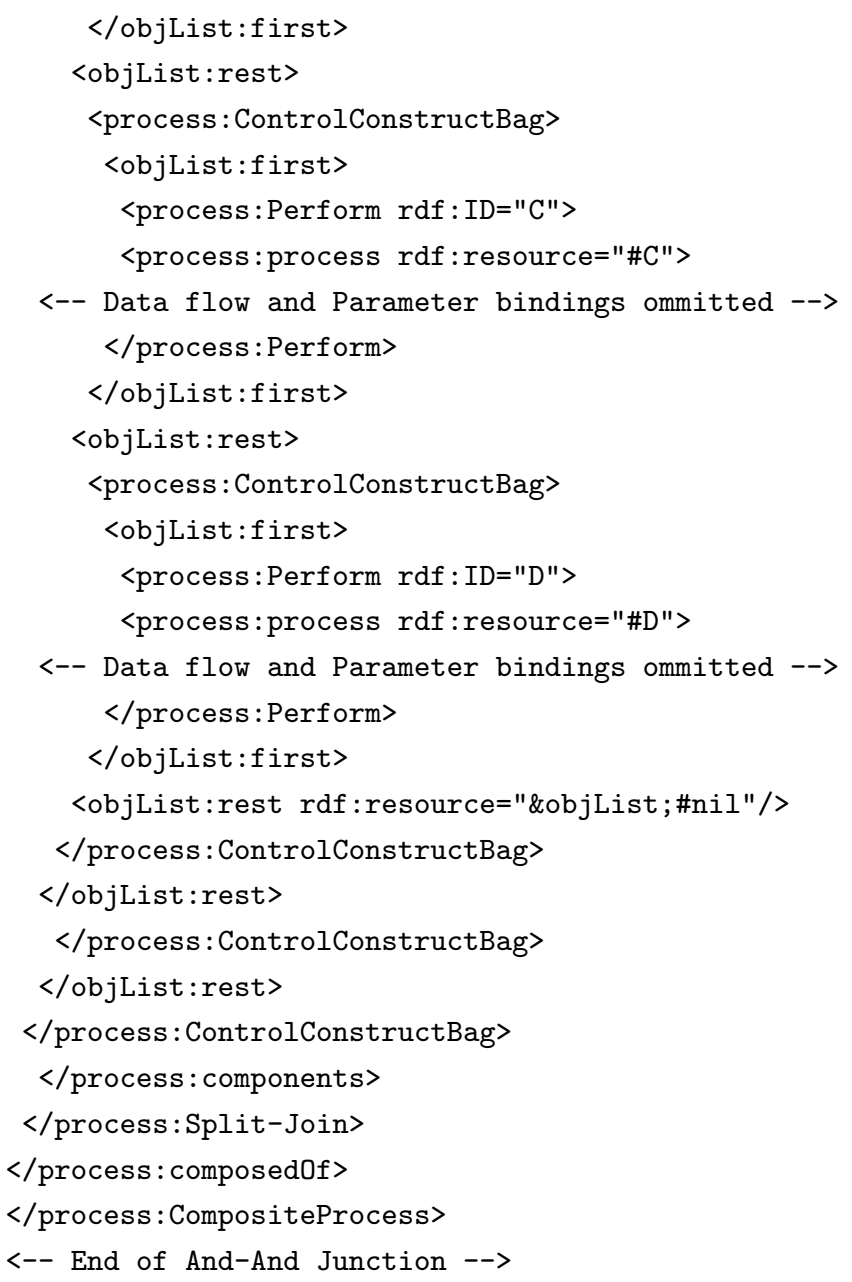

\section{Example 2. A Complex Layered Model}

FBPML formal notation for process model in Figure 12:

01 start('A').

02 activity(08, 'Xor-Xor', Trig1, Precond1, Postcond1, Act1, 'Desc2').

03 junction('Xor', 'A', ['And-And', 'D']).

04 junction('Xor', ['And-And', 'D'], 'E').

05 primitive_activity(001, 'E', Trig2, Precond2, Postcond2, Act2, 'Desc2').

06 finish('E').

07 activity(09, 'And-And', Trig3, Precond3, Postcond3, Act3, 'Desc3').

08 junction('And', 'J1', ['B', 'C']).

09 junction('And', ['B', 'C'], 'J2').

10 primitive_activity(002, 'A', Trig4, Precond4, Postcond4, Act4, 'Desc4').

11 primitive_activity(003, 'B', Trig5, Precond5, Postcond5, Act5, 'Desc5').

12 primitive_activity(004, 'C', Trig6, Precond6, Postcond6, Act6, 'Desc6').

13 primitive_activity(005, 'D', Trig7, Precond7, Postcond7, Act7, 'Desc7').

Assumptions and Interpretations:

- Ordering of junctions are from top-most (outer) to down-most (inner). For example the Xor-Xor definition (lines 3-4) appears before the And-And definition (lines 8-9). 


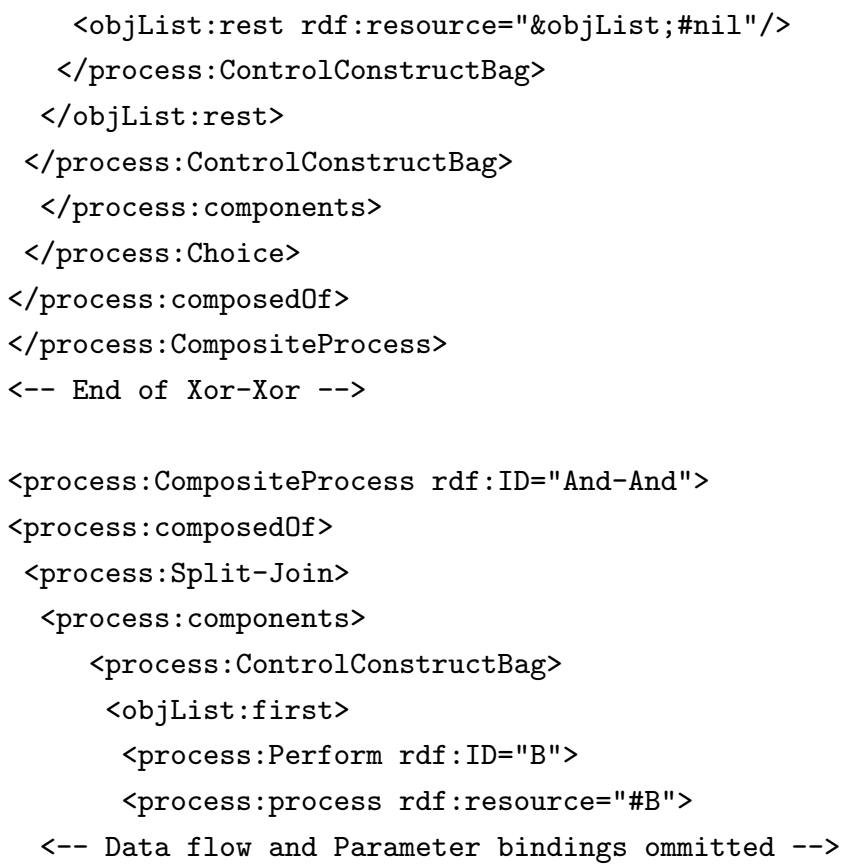

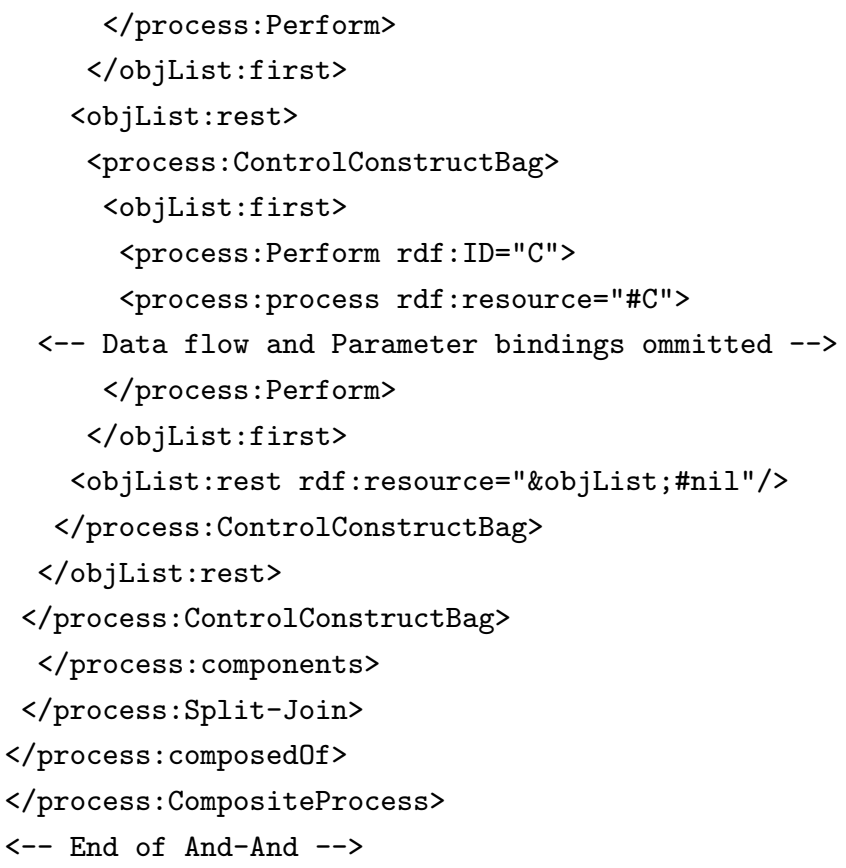

\section{Implementation of Process Model Translator}

A process model translator was developed using SICStus Prolog 3.10.1 on (Red Hat) Linux 9. The design was based on parsing first-order logic (Horn) clauses into hierarchical OWL and RDF tree-like tags. The overall architecture diagram is given by Figure 14. The general algorithm was based on process decomposition as outlined in the previous section. The initial implementation was based on specific simple procedures which were built up incrementally as the complexity of the process model increased. The main aim of the process model translator was to cater for any process model described in FBPML PL to be converted into OWL syntax. As pointed out in the previous section, the process model translation does not encompass all the possible primitives and process constructs, and is therefore limited. Hence the system was implemented to perform the translation as closely, accurately and directly as possible, taking into account some viable assumptions and interpretations.

The system implemented encompassed six types of translations, namely simple Sequence, And-Split, And-Joint, Xor-Split, And-And combination and complex layered model with any of these combinations. The algorithm provided explains the methodology adhered by the main predicate of the program. A process model is parsed and recognised as one of the six mentioned above in lines 6-13. Each junction will be further parsed to determine the nature of the junction; be it a split or a joint. Decompostion will take place until an atomic process is encountered.

\section{Evaluation}

The conceptual mapping framework and process model translator implementation demonstrated that all of the FBPML data model components could be translated directly to OWL, while the process model components, in particular the junctions, could only be partially (or not) translated as FBPML has a larger set of modelling primitives. A constituent of a FBPML process model that could not be translated is recorded in the OWL-S comment construct after the automatic translation is completed.

The analysis suggests that a full translation between all FBPML process models and OWL-S is not possible. This is mainly due to the fact that there are some elements that exist in FBPML but not in OWL-S such as Trigger, 


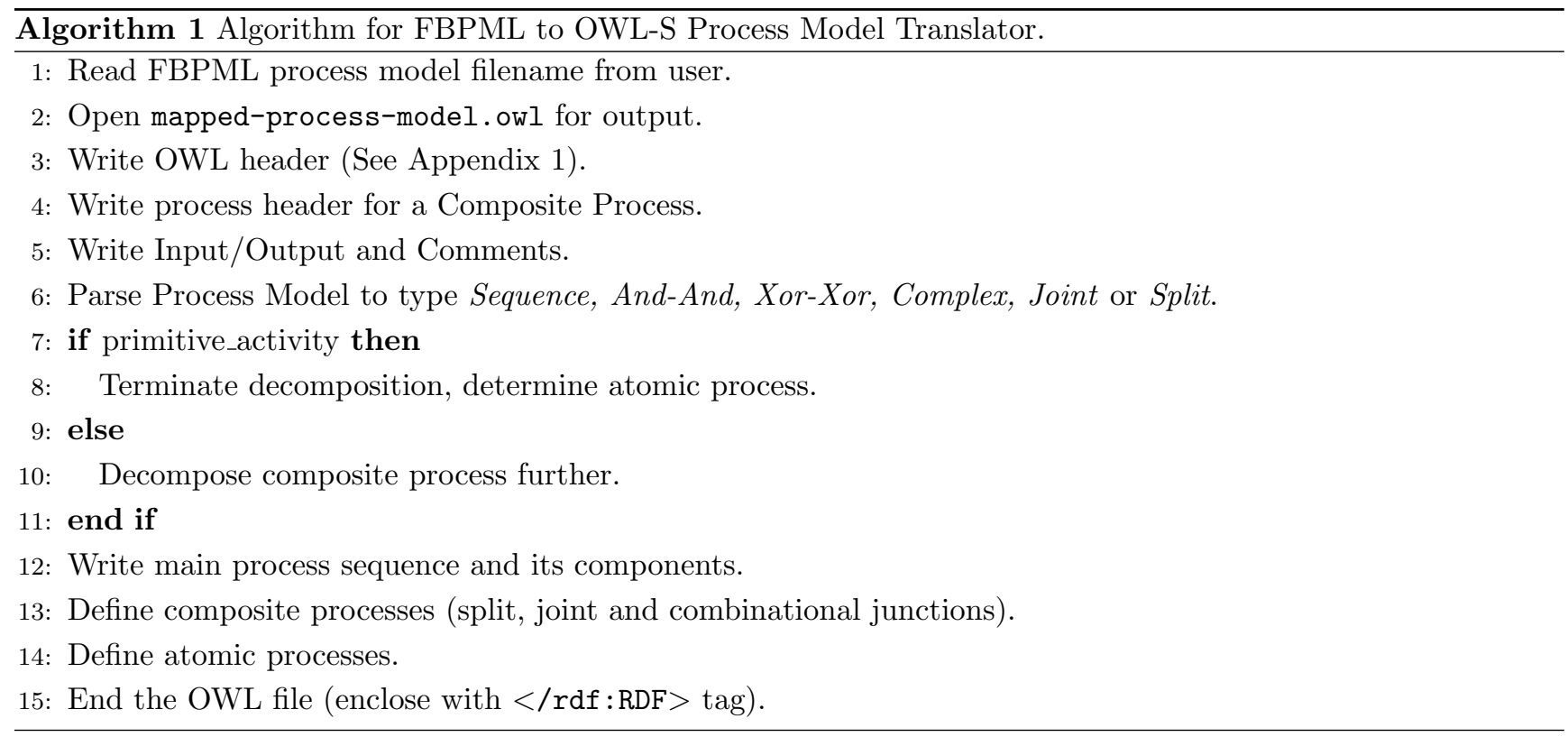

Or, time points and synchronisation of time points (See Table 4). The implications of such missing elements could be severe from a business processing point of view. Consider a business scenario whereby the service requester (customer) wishes to make a payment for a service. The options for payment methods are credit card, cash or cheque. The FBPML process model is given in Figure 15 whereby the customer may choose to pay by one or more of these methods, allowing for partial payments using several methods. Without the provision of Or within OWL-S, the customer is only given one option of payment using OWL-S in such scenarios, since this process model would be most closely represented using the Choice construct (which is equivalent to Xor), making it rather limited.

Another reason a full translation cannot be achieved is due to lack of knowledge about an aspect that is still in progress. OWL doesn't support representation of business rules and such rules must be described using a different logical formalism, e.g. SWRL. This leads to the inability of representing some types of business process models in OWL-S.

Additional problems will arise if partially overlapping loops exist in the FBPML process model as described in [12]. When this happens, the process model may not be decomposed in constructs specified by OWL-S, thus causing mapping problems. However, the modelers of OWL-S expect that most OWL-S process models to be loop-free.

Thus, the mapping between FBPML and OWL-S is very challenging. The implementation of the process model translator, although limited, is able to decompose and map the sequences and combination junctions in a methodical manner. The essence of the analysis is that a much thorough understanding for both languages has been gained and this experience can contribute to other business process modellers wishing to use Semantic Web services based on OWL-S.

\section{Conclusions}

A lightweight conceptual mapping framework has been demonstrated between two formal languages, FBPML and OWL-S. The former is traditionally used in the context of business process modelling and the latter in the domain of Semantic Web services. An automatic system for the process model translation has also been implemented although not all the constructs in FBPML could be translated to OWL-S. Furthermore, the specifications of some 
aspects of OWL-S are still in progress and hence the translation could not be performed.

A complete formalism for rules and conditions within OWL would allow for some of the gaps between FBPML and OWL-S to be filled. As the future for OWL-S remains unclear, current effort towards converging OWL-S with WSMO could be a positive step towards the development of a stronger and more stable global standard for Semantic Web services. It would also be worthwhile to perform a translation between FBPML and WSMO in due course.

The conceptual mapping exercise and implementation have brought to light some vital differences between the constructions of the languages in the EM and Semantic Web services worlds. It is interesting to investigate the relationships between traditional and cutting edge technologies so that both fields could benefit from each other.

As a closure, narrowing the gap between business process technologies and Semantic Web services has opened a window of opportunity for the more established BPM methods to be utilised by the evolving Semantic Web technologies. It is hoped that the growth of potential Semantic Web standards such as OWL-S could be strengthened and enriched by manipulating more mature technologies such as BPM methods.

\section{References}

[1] AKT. Advanced Knowledge Technologies IRC Project Technology Showcase. Aberdeen, Edinburgh, Open, Sheffield, Southampton Universities, 2002. http://www.aktors.org.

[2] G. Antoniou and F. van Harmelen. A Semantic Web Primer. MIT Press, Cambridge, MA, USA, 2004.

[3] A. Arkin. Business Process Modelling Language (BPML). 2002.

[4] M. A. Aslam, S. Auer, and J. Shen. From BPEL4WS Process Model to Full OWL-S Ontology. In Third European Semantic Web Conference 2006 (ESWC06), 2006.

[5] T. Berners-Lee, J. Hendler, and O. Lassila. The Semantic Web. Scientific American, 284(5), May 2001.

[6] BPEL4WS. Business Process Execution Language for Web Services Version 1.1. IBM, BEA Systems, Microsoft, SAP AG, Siebel Systems, 2003. http://www-128.ibm.com/developerworks/library/specification/ws-bpel/.

[7] Y.-H. Chen-Burger. Informal Semantics for the FBPML Data Language. Technical report, Informatics Research Reports, School of Informatics, University of Edinburgh, 2002.

[8] Y.-H. Chen-Burger and D. Robertson. Automating Business Modelling: A Guide to Using Logic to Represent Informal Methods and Support Reasoning. Book Series of Advanced Information and Knowledge Processing, Springer Ver-Lag, 2005.

[9] Y.-H. Chen-Burger, A. Tate, and D. Robertson. Enterprise Modelling: A Declarative Approach for FBPML. In Proceedings European Conference of Artificial Intelligence, Knowledge Management and Organisational Memories Workshop, 2002.

[10] E. Christensen, F. Curbera, G. Meredith, and S. Weerawarana. Web Services Description Language (WSDL) 1.1. W3C Note, 2001.

[11] D. Fensel and C. Bussler. The Web Service Modeling Framework WSMF. Electronic Commerce Research and Applications, 2002.

[12] L. Guo, Y.-H. Chen-Burger, and D. Robertson. Mapping a Business Process Model to a Semantic Web Service Model. In Third IEEE International Conference on Web Services (ICWS04), 2004.

[13] M. Hepp, F. Leymann, J. Domingue, A. Wahler, and D. Fensel. Semantic Business Process Management: A Vision Towards Using Semantic Web Services for Business Process Management. ICEBE, 0:535-540, 2005.

[14] I. Horrocks, P. F. Patel-Schneider, H. Boley, S. Tabet, B. Grosof, and M. Dean. SWRL: A Semantic Web Rule Language Combining OWL and RuleML. National Research Council of Canada, Network Inference, and Stanford University, 2004.

[15] G. Klyne and J. Carroll, editors. Resource Description Framework (RDF): Concepts and Abstract Syntax. W3C Recommendation, 2004.

[16] R. Lara, D. Roman, A. Polleres, and D. Fensel. A Conceptual Comparison of WSMO and OWL-S. In ECOWS, pages 254-269, 2004. 
[17] H. Lausen, J. de Bruijn, A. Polleres, and D. Fensel. WSML - a Language Framework for Semantic Web Services. In Position Paper for the W3C rules workshop, Washington DC, USA, 2005.

[18] D. Martin, editor. OWL-S Semantic Markup for Web Services, Pre-Release 1.2. World Wide Web Consortium (W3C), 2006. http://www.ai.sri.com/daml/services/owl-s/1.2/ (Temporary location at SRI).

[19] R. Mayer, C. Menzel, M. Painter, P. Witte, T. Blinn, and B. Perakath. Information Integration for Concurrent Engineering (IICE) IDEF3 Process Description Capture Method Report. Knowledge Based Systems Inc., Sept 1995.

[20] D. McGuinness and F. van Harmelen. OWL Web Ontology Language. World Wide Web Consortium (W3C), 2004. http://www.w3.org/TR/owl-features/.

[21] G. Nadarajan and Y.-H. Chen-Burger. An Ontology-Based Conceptual Mapping Framework for Translating FBPML to the Web Services Ontology. CCGRID06, 0:158-165, 2006.

[22] G. Nadarajan and Y.-H. Chen-Burger. Mapping Fundamental Business Process Modelling Language to OWL-S. In Hellenic Conference in AI (SETN06), pages 563-566, 2006.

[23] M. Paolucci, N. Srinivasan, and K. Sycara. Expressing WSMO mediators in OWL-S. In Semantic Web Services Workshop at ISWC, 2004.

[24] W. Reisig. Petri Nets, an Introduction. In EATCS, Monographs on Theoretical Computer Science, 1985.

[25] D. Roman, H. Lausen, and U. Keller, editors. Web Service Modeling Ontology (WSMO). WSMO Final Draft, 2005. http://www.wsmo.org/TR/d2/v1.2/.

[26] J. Rumbaugh, I. Jacobson, and G. Booch. The Unified Modeling Language Reference Manual. Addison-Wesley, 1998.

[27] F. Scharffe and J. de Bruijn. A Language to Specify Mappings between Ontologies. In Proceedings of the Internet Based Systems IEEE Conference (SITIS05), Yandoué, Cameroon, November 2005.

[28] C. Schlenoff, A. Knutila, and S. Ray, editors. Proceedings of the First Process Specification Language (PSL) Roundtable. National Institute of Standards and Technology, Gaithersburg, MD, 1997. http://www.nist.gov/psl/.

[29] J. Scicluna, R. Lara, A. Polleres, and H. Lausen. Formal Mapping and Tool to OWL-S. WSMO Working Draft, Dec. 2004.

[30] J. Shen, Y. Yang, C. Wan, and C. Zhu. From BPEL4WS to OWL-S: Integrating E-Business Process Descriptions. In SCC '05: Proceedings of the 2005 IEEE International Conference on Services Computing, pages 181-190, Washington, DC, USA, 2005. IEEE Computer Society.

[31] The Workflow Management Coalition, Dec 2003. http://www.wfmc.org.

\section{Appendix}

\section{A.1 OWL header file}

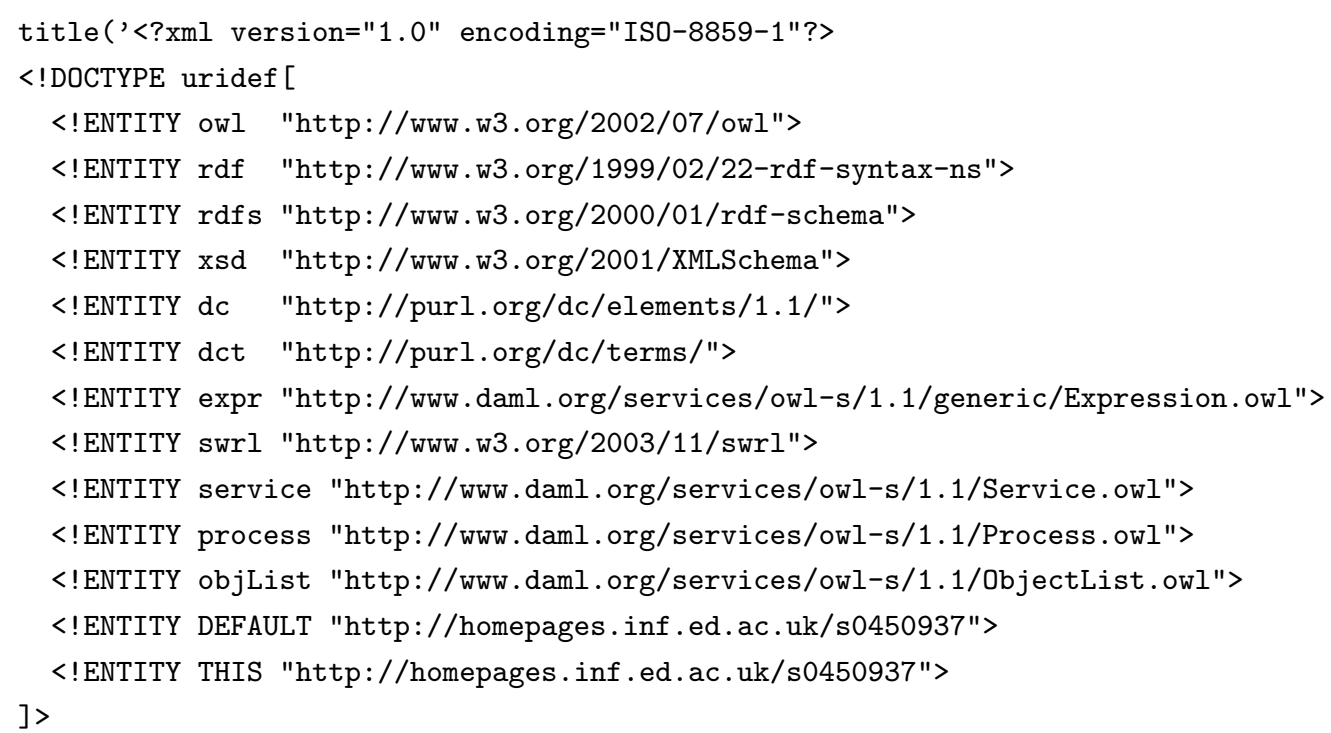




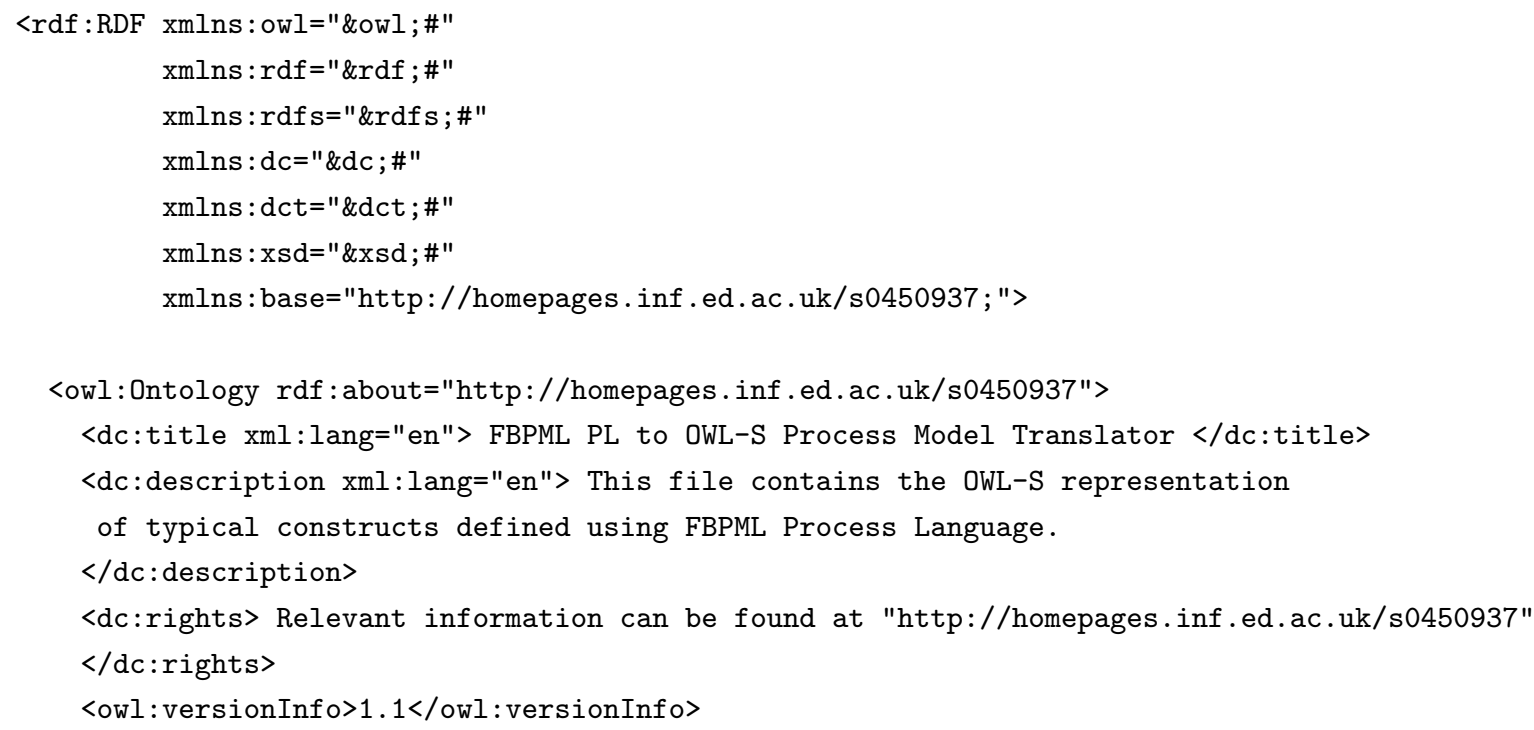

\section{List of Figures}

1 FBPML visual notation. . . . . . . . . . . . . . . . . . . . . 26

2 The FBPML to OWL-S Conceptual Mapping Framework . . . . . . . . . . . . . . . . . . . 26

3 Ontology notation for FBPML's data model. . . . . . . . . . . . . . . . . . . 26

4 Ontology notation for OWL-S's data model. . . . . . . . . . . . . . . . . . . . . . . . 27

5 Main FBPML junctions. . . . . . . . . . . . . . . . . . . . . . . 27

6 Ontology notation for FBPML process model. . . . . . . . . . . . . . . . . . . . 27

7 OWL-S process model ontology [18] . . . . . . . . . . . . . . . . . . . . . . . 28

8 Process diagram of a sequence of activities in FBPML . . . . . . . . . . . . . . . . . . 28

$9 \quad$ FBPML And-Joint Junction (with barrier synchronisation). . . . . . . . . . . . . . . . . . . 28

10 FBPML And-And Junction. . . . . . . . . . . . . . . . . . . . . . . . . . . 28

11 Decomposition of FBPML process model containing the And-And Junction. . . . . . . . . . . . . . 28

12 A complex FBPML process model. . . . . . . . . . . . . . . . . . . . . . . . 29

13 Decomposition of the complex process model. . . . . . . . . . . . . . . . . . . . . 29

14 An Architectural Overview of the FBPML to OWL-S Mapping Engine. . . . . . . . . . . . . . . . 29

15 A Business Scenario for 'Making a Payment' process. . . . . . . . . . . . . . . . . . . . . 29 


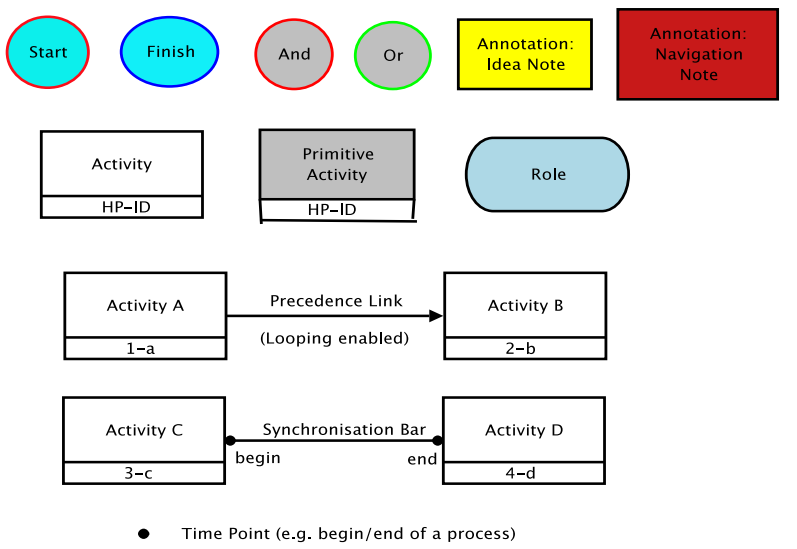

Figure 1. FBPML visual notation.

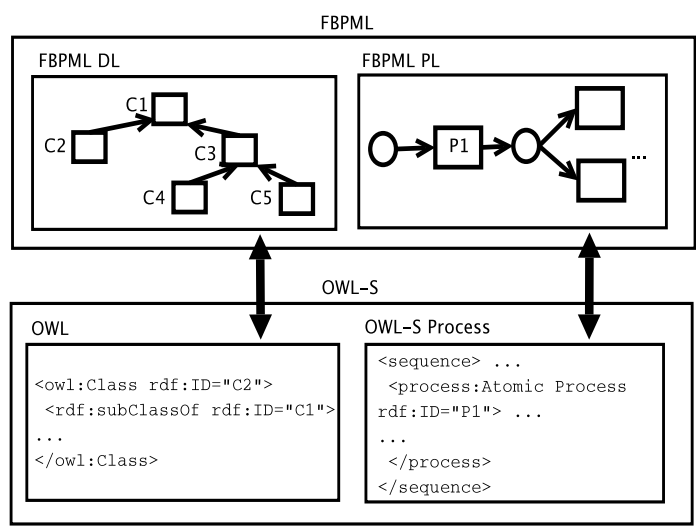

Figure 2. The FBPML to OWL-S Conceptual Mapping Framework

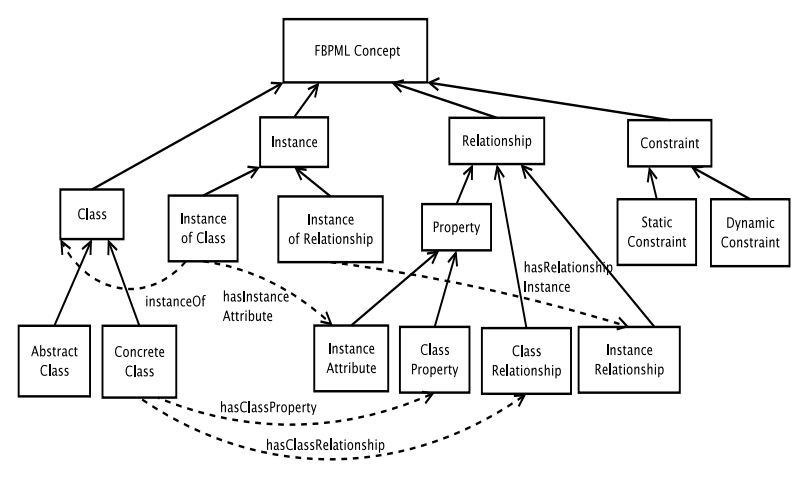

Figure 3. Ontology notation for FBPML's data model. 


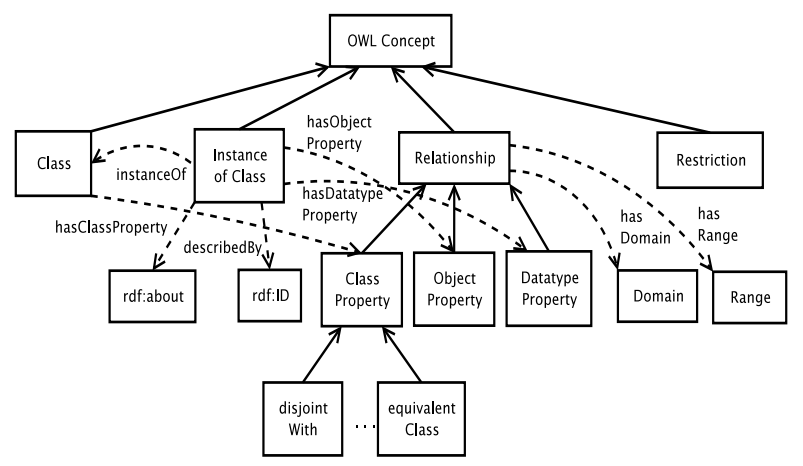

Figure 4. Ontology notation for OWL-S's data model.
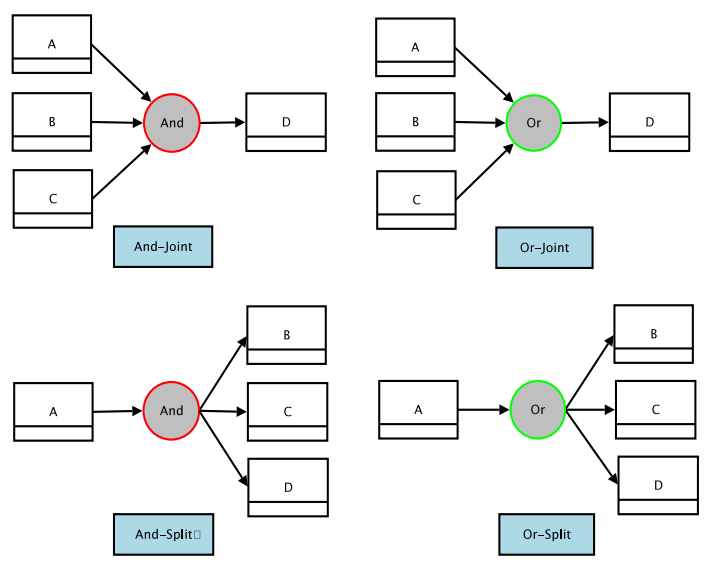

Figure 5. Main FBPML junctions.

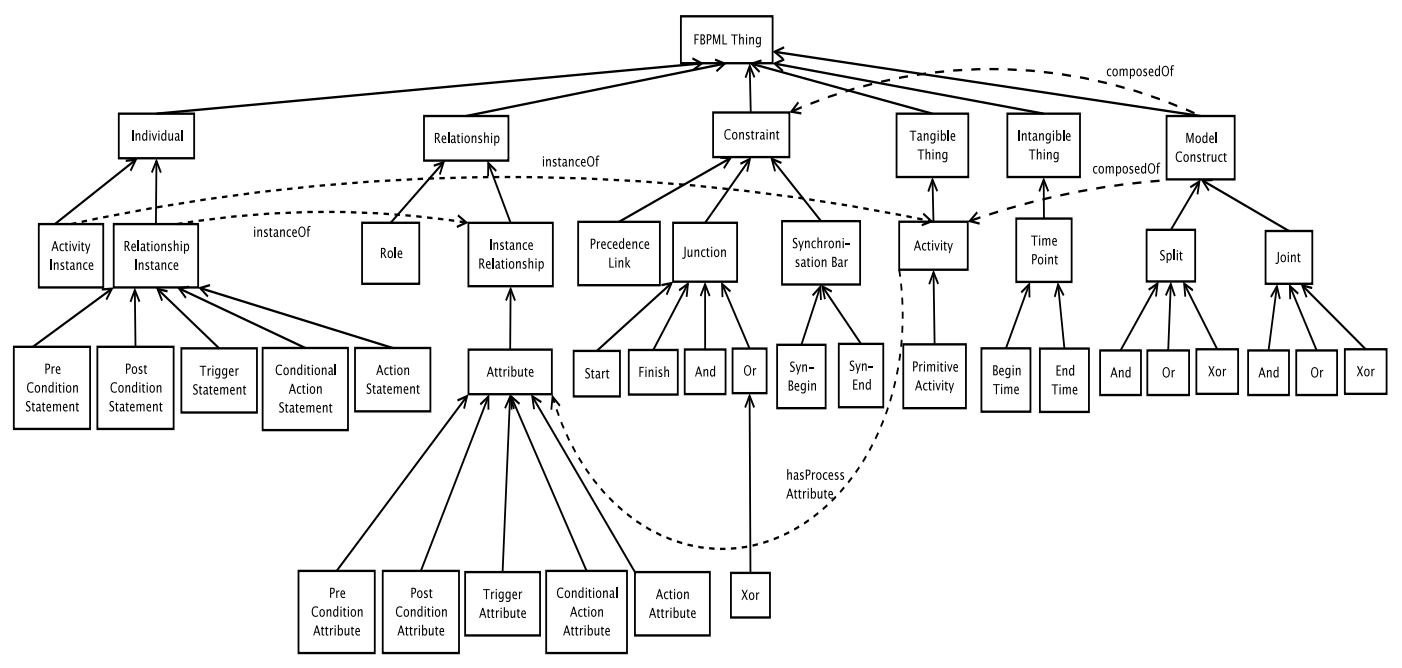

Figure 6. Ontology notation for FBPML process model. 


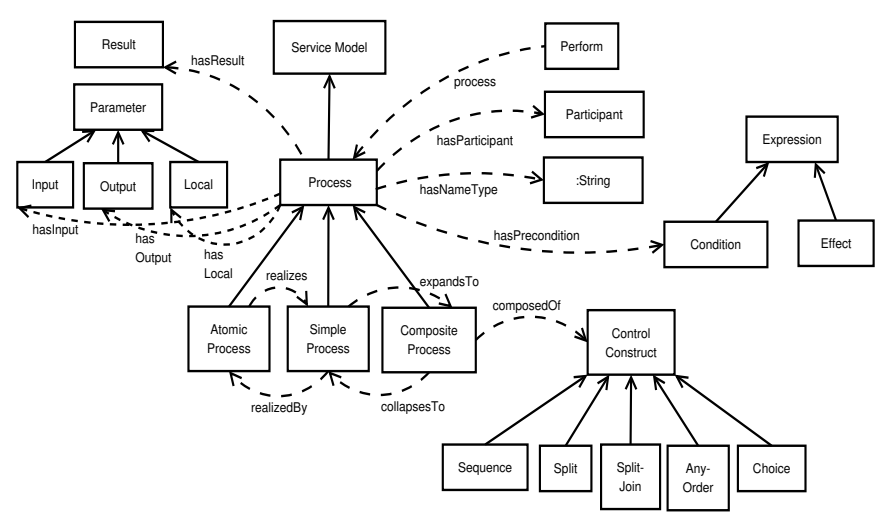

Figure 7. OWL-S process model ontology [18].

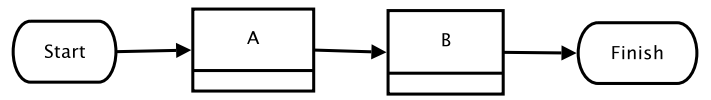

Figure 8. Process diagram of a sequence of activities in FBPML.

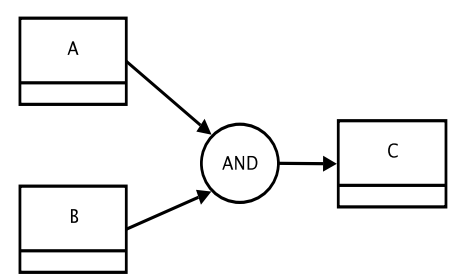

Figure 9. FBPML And-Joint Junction (with barrier synchronisation).

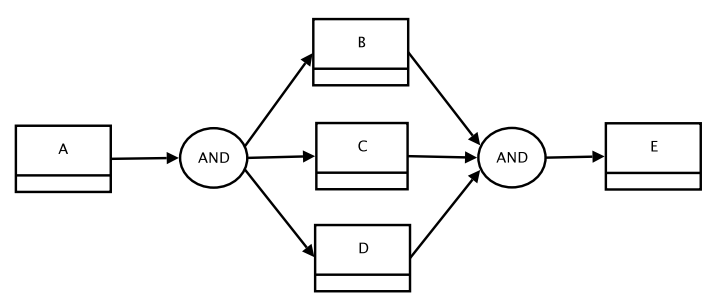

Figure 10. FBPML And-And Junction.

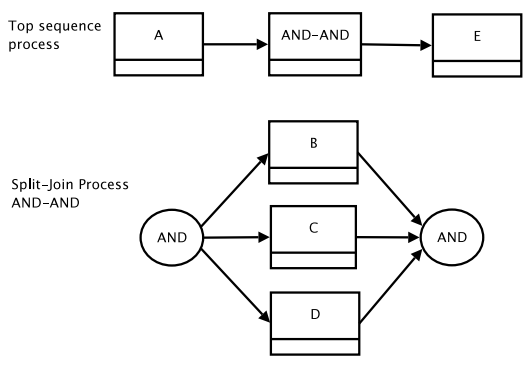

Figure 11. Decomposition of FBPML process model containing the And-And Junction. 


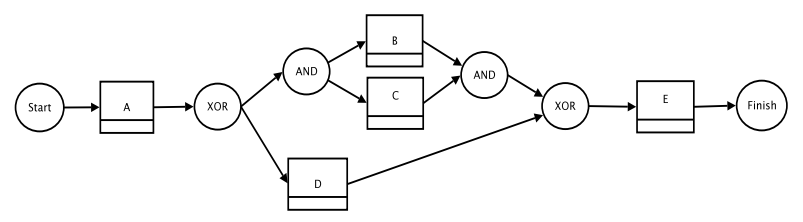

Figure 12. A complex FBPML process model.

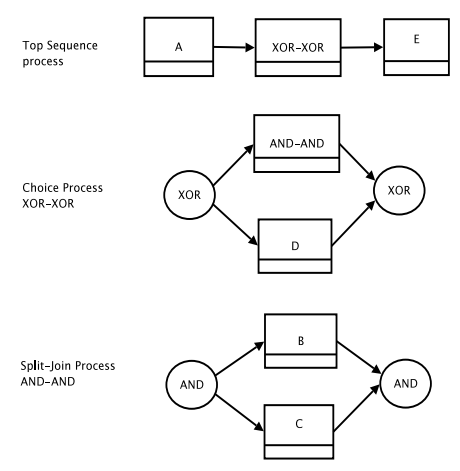

Figure 13. Decomposition of the complex process model.

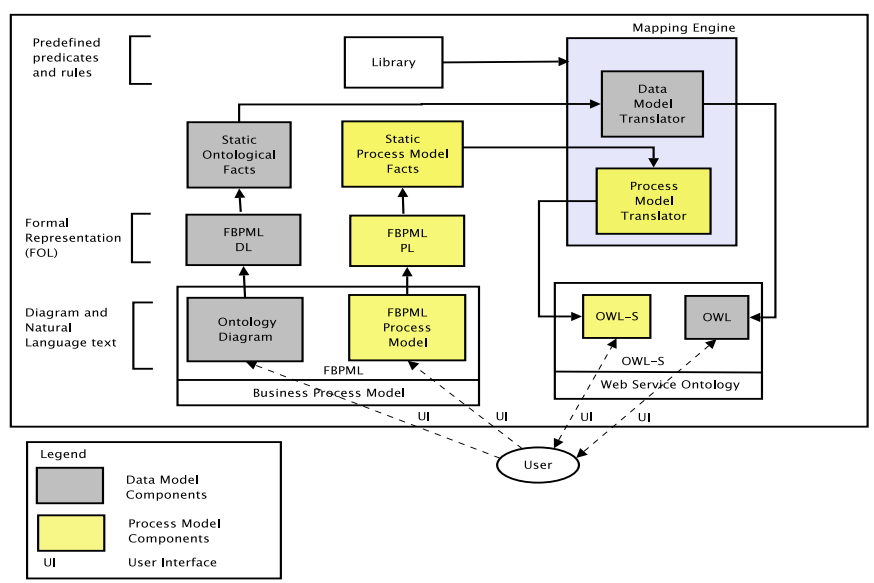

Figure 14. An Architectural Overview of the FBPML to OWL-S Mapping Engine.

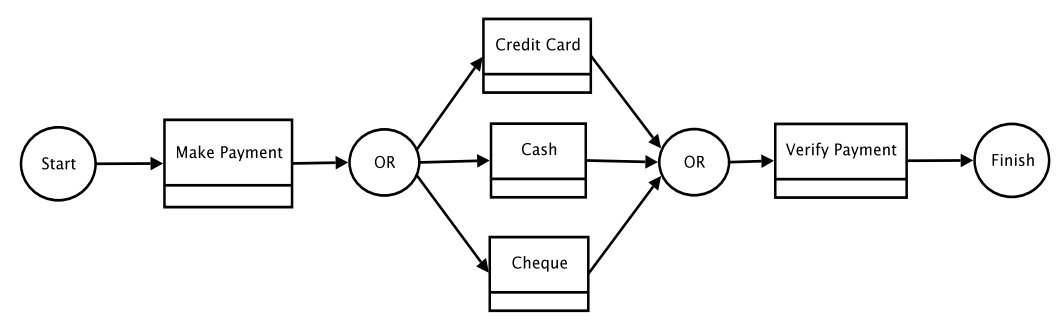

Figure 15. A Business Scenario for 'Making a Payment' process. 